R. H. Tütüncü, K. C. Toh, · M. J. Todd

\title{
Solving semidefinite-quadratic-linear programs using SDPT3 *
}

Received: March 19, 2001 / Accepted: January 18, 2002

Published online: October 9, 2002 - (C)Springer Verlag 2002

Abstract. This paper discusses computational experiments with linear optimization problems involving semidefinite, quadratic, and linear cone constraints (SQLPs). Many test problems of this type are solved using a new release of SDPT3, a MATLAB implementation of infeasible primal-dual path-following algorithms. The software developed by the authors uses Mehrotratype predictor-corrector variants of interior-point methods and two types of search directions: the HKM and NT directions. A discussion of implementation details is provided and computational results on problems from the SDPLIB and DIMACS Challenge collections are reported.

\section{Introduction}

Conic linear optimization problems can be expressed in the following standard form:

$$
\begin{aligned}
& \min \langle c, x\rangle \\
& \text { s.t. } \quad\left\langle a_{k}, x\right\rangle=b_{k}, \quad k=1, \ldots, m, \\
& \quad x \in K
\end{aligned}
$$

where $K$ is a closed, convex pointed cone in a finite dimensional inner product space endowed with an inner product $\langle\cdot, \cdot\rangle$. By choosing $K$ to be the semidefinite, quadratic (second-order), and linear cones respectively, one obtains the wellknown special cases of semidefinite, second-order cone, and linear programming problems. Recent years have seen a dramatic increase in the number of subclasses of conic optimization problems that can be solved efficiently by interior-point methods. In addition to the ongoing theoretical work that derived convergence guarantees and convergence rates for such algorithms, many groups of researchers

R. H. Tütüncü: Department of Mathematical Sciences, Carnegie Mellon University, Pittsburgh, PA 15213, USA. e-mail: reha@andrew.cmu.edu. Research supported in part by NSF through grant CCR-9875559.

K. C. Toh: Department of Mathematics, National University of Singapore, 10 Kent Ridge Crescent, Singapore 119260. e-mail: mattohkc@math.nus.edu.sg. Research supported in part by the Singapore-MIT Alliance.

M. J. Todd: School of Operations Research and Industrial Engineering, Cornell University, Ithaca, New York 14853, USA. e-mail: miketodd@cs. cornell.edu. Research supported in part by NSF through grant DMS-9805602 and ONR through grant N00014-96-1-0050.

Mathematics Subject Classification (1991): 90C05, 90C22

* Copyright (C) by Springer-Verlag. Mathematical Programming 95 (2003), 189-217. 
have also implemented these algorithms and developed public domain software packages that are capable of solving conic optimization problems of ever increasing size and diversity. This paper discusses the authors' contribution to this effort through the development of the software SDPT3. Our earlier work on SDPT3 is presented in $[22,25]$.

The current version of SDPT3, version 3.0, can solve conic linear optimization problems with inclusion constraints for the cone of positive semidefinite matrices, the second-order cone, and/or the polyhedral cone of nonnegative vectors. In other words, we allow $K$ in (1) to be a Cartesian product of cones of positive semidefinite matrices, second-order cones, and the nonnegative orthant. We use the following standard form of such problems, henceforth called SQLP problems:

$$
\begin{aligned}
& (P) \min \quad \sum_{j=1}^{n_{s}}\left\langle c_{j}^{s}, x_{j}^{s}\right\rangle+\sum_{i=1}^{n_{q}}\left\langle c_{i}^{q}, x_{i}^{q}\right\rangle+\left\langle c^{l}, x^{l}\right\rangle \\
& \text { s.t. } \sum_{j=1}^{n_{s}}\left(A_{j}^{s}\right)^{T} \operatorname{svec}\left(x_{j}^{s}\right)+\sum_{i=1}^{n_{q}}\left(A_{i}^{q}\right)^{T} x_{i}^{q}+\left(A^{l}\right)^{T} x^{l}=b, \\
& x_{j}^{s} \in K_{s}^{s_{j}} \forall j, \quad x_{i}^{q} \in K_{q}^{q_{i}} \forall i, \quad x^{l} \in K_{l}^{n_{l}} .
\end{aligned}
$$

Here, $c_{j}^{s}, x_{j}^{s}$ are symmetric matrices of dimension $s_{j}$ and $K_{s}^{s_{j}}$ is the cone of positive semidefinite symmetric matrices of the same dimension. Similarly, $c_{i}^{q}$, $x_{i}^{q}$ are vectors in $\mathbb{R}^{q_{i}}$ and $K_{q}^{q_{i}}$ is the second-order cone defined by $K_{q}^{q_{i}}:=$ $\left\{x \in \mathbb{R}^{q_{i}}: x_{1} \geq\left\|x_{2: q_{i}}\right\|\right\}$. Finally, $c^{l}, x^{l}$ are vectors of dimension $n_{l}$ and $K_{l}^{n_{l}}$ is the cone $\mathbb{R}_{+}^{n_{l}}$. In the notation above, $A_{j}^{s}$ denotes the $\bar{s}_{j} \times m$ matrix with $\bar{s}_{j}=s_{j}\left(s_{j}+1\right) / 2$ whose columns are obtained using the svec operator from $m$ symmetric $s_{j} \times s_{j}$ constraint matrices corresponding to the $j$ th semidefinite block $x_{j}^{s}$. (Here, for a symmetric matrix $x$ of order $s, \operatorname{svec}(x):=$ $\left(x_{11}, \sqrt{2} x_{12}, x_{22}, \sqrt{2} x_{13}, \sqrt{2} x_{23}, x_{33}, \ldots\right)^{T} \in \mathbb{R}^{s(s+1) / 2}$, where the $\sqrt{2}$ is to make the operation an isometry.) The matrices $A_{i}^{q}$ 's are $q_{i} \times m$ dimensional constraint matrices corresponding to the $i$ th quadratic block $x_{i}^{q}$, and $A^{l}$ is the $l \times m$ dimensional constraint matrix corresponding to the linear block $x^{l}$. The notation $\langle p, q\rangle$ denotes the standard inner product in the appropriate space.

The software also solves the dual problem associated with the problem above:

$$
\begin{aligned}
\text { (D) } \max & b^{T} y \\
\text { s.t. } & A_{j}^{s} y+z_{j}^{s}=c_{j}^{s}, \quad j=1 \ldots, n_{s}, \\
& A_{i}^{q} y+z_{i}^{q}=c_{i}^{q}, \quad i=1 \ldots, n_{q}, \\
& A^{l} y+z^{l}=c^{l}, \\
z_{j}^{s} \in K_{s}^{s_{j}} & \forall j, \quad z_{i}^{q} \in K_{q}^{q_{i}} \forall i, \quad z^{l} \in K_{l}^{n_{l}} .
\end{aligned}
$$

This package is written in MATLAB version 5.3 and is compatible with MATLAB version 6.0. It is available from the internet sites:

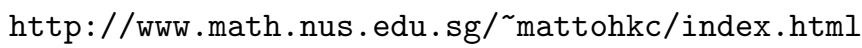

$$
\begin{aligned}
& \text { http://www .math.cmu.edu/ reha/sdpt3.html }
\end{aligned}
$$

This software package was originally developed to provide researchers in semidefinite programming with a collection of reasonably efficient and robust 
algorithms that can solve general SDPs with matrices of dimensions of the order of a hundred. The current release, version 3.0, expands the family of problems solvable by the software in two dimensions. First, this version is much faster than the previous release [25], especially on large sparse problems, and consequently can solve much larger problems. Second, the current release can also directly solve problems that have second-order cone constraints - with the previous version it was necessary to convert such constraints to semidefinite cone constraints.

In this paper, the vector 2-norm and Frobenius norm are denoted by $\|\cdot\|$ and $\|\cdot\|_{F}$, respectively. In the next section, we discuss the algorithm used in the software and several computational details. Section 3 describes the initial iterates generated by our software while Section 4 briefly describes its options, some implementation details, and its data storage scheme. In Section 5, we present and comment on the results of our computational experiments with our software on problems from the SDPLIB and DIMACS libraries. Section 6 contains a short conclusion.

\section{A primal-dual infeasible-interior-point algorithm}

The algorithm implemented in SDPT3 is a primal-dual interior-point algorithm that uses the path-following paradigm. In each iteration, we first compute a predictor search direction aimed at decreasing the duality gap as much as possible. After that, the algorithm generates a Mehrotra-type corrector step [14] with the intention of keeping the iterates close to the central path. However, we do not impose any neighborhood restrictions on our iterates. ${ }^{1}$ Initial iterates need not be feasible - the algorithm tries to achieve feasibility and optimality of its iterates simultaneously.

It should be noted that our implementation allows the user to switch to a primal-dual path-following algorithm that does not use corrector steps and sets a centering parameter to be used in such a framework. The choices we make on the parameters used by the algorithm are based on minimizing either the number of iterations or the CPU time of the linear algebra involved in computing the Schur complement matrix and its Cholesky factorization. What follows is a pseudo-code for the algorithm we implemented. Note that this description makes references to later parts of this section where many details related to the algorithm are explained.

Algorithm IPC. Suppose we are given an initial iterate $\left(x^{0}, y^{0}, z^{0}\right)$ with $x^{0}, z^{0}$ strictly satisfying all the conic constraints. Decide on the type of search direction to use. Set $\gamma^{0}=0.9$. Choose a value for the parameter expon used in e.

For $k=0,1, \ldots$

1 This strategy works well on most problems we tested. However, it should be noted that the occasional failure of the software on problems with poorly chosen initial iterates is likely due to the lack of a neighborhood enforcement in the algorithm. 
(Let the current and the next iterate be $(x, y, z)$ and $\left(x^{+}, y^{+}, z^{+}\right)$respectively. Also, let the current and the next step-length parameter be denoted by $\gamma$ and $\gamma^{+}$respectively.)

- Set $\mu=\langle x, z\rangle / n$, and

$$
\text { relgap }=\frac{\langle x, z\rangle}{1+\max \left(|\langle c, x\rangle|,\left|b^{T} y\right|\right)}, \quad \phi=\max \left(\frac{\left\|r_{p}\right\|}{1+\|b\|}, \frac{\left\|R_{d}\right\|}{1+\|c\|}\right) .
$$

Stop the iteration if the infeasibility measure $\phi$ and the relative duality gap (relgap) are sufficiently small.

- (Predictor step)

Solve the linear system (10), with $\sigma=0$ in the right-side vector (12). Denote the solution of (4) by $(\delta x, \delta y, \delta z)$. Let $\alpha_{p}$ and $\beta_{p}$ be the step-lengths defined as in (33) and (34) with $\Delta x, \Delta z$ replaced by $\delta x, \delta z$, respectively.

- Take $\sigma$ to be

$$
\sigma=\min \left(1,\left[\frac{\left\langle x+\alpha_{p} \delta x, z+\beta_{p} \delta z\right\rangle}{\langle x, z\rangle}\right]^{e}\right),
$$

where the exponent $e$ is chosen as follows:

$$
e= \begin{cases}\max \left[\text { expon, } 3 \min \left(\alpha_{p}, \beta_{p}\right)^{2}\right] & \text { if } \mu>10^{-6}, \\ \text { expon } & \text { if } \mu \leq 10^{-6} .\end{cases}
$$

- (Corrector step)

Solve the linear system (10) with $R_{c}$ in the the right-hand side vector (12) replaced by

$$
\begin{aligned}
& \tilde{R}_{c}^{s}=\operatorname{svec}\left[\sigma \mu I-H_{P}\left(\operatorname{smat}\left(x^{s}\right) \operatorname{smat}\left(z^{s}\right)\right)-H_{P}\left(\operatorname{smat}\left(\delta x^{s}\right) \operatorname{smat}\left(\delta z^{s}\right)\right)\right] \\
& \tilde{R}_{c}^{q}=\sigma \mu e^{q}-T_{G}\left(x^{q}, z^{q}\right)-T_{G}\left(\delta x^{q}, \delta z^{q}\right) \\
& \tilde{R}_{c}^{l}=\sigma \mu e^{l}-\operatorname{diag}\left(x^{l}\right) z^{l}-\operatorname{diag}\left(\delta x^{l}\right) \delta z^{l} .
\end{aligned}
$$

Denote the solution of (4) by $(\Delta x, \Delta y, \Delta z)$.

- Update $(x, y, z)$ to $\left(x^{+}, y^{+}, z^{+}\right)$by

$$
x^{+}=x+\alpha \Delta x, \quad y^{+}=y+\beta \Delta y, \quad z^{+}=z+\beta \Delta z,
$$

where $\alpha$ and $\beta$ are computed as in (33) and (34) with $\gamma$ chosen to be $\gamma=0.9+0.09 \min \left(\alpha_{p}, \beta_{p}\right)$.

- Update the step-length parameter by

$$
\gamma^{+}=0.9+0.09 \min (\alpha, \beta)
$$

\subsection{The search direction}

To simplify discussion, we introduce the following notation, which is also consistent with the internal data representation in SDPT3:

$$
A^{s}=\left[\begin{array}{c}
A_{1}^{s} \\
\vdots \\
A_{n_{s}}^{s}
\end{array}\right], \quad A^{q}=\left[\begin{array}{c}
A_{1}^{q} \\
\vdots \\
A_{n_{q}}^{q}
\end{array}\right] .
$$

Similarly, we define

$$
x^{s}=\left[\begin{array}{c}
\operatorname{svec}\left(x_{1}^{s}\right) \\
\vdots \\
\operatorname{svec}\left(x_{n_{s}}^{s}\right)
\end{array}\right], \quad x^{q}=\left[\begin{array}{c}
x_{1}^{q} \\
\vdots \\
x_{n_{q}}^{q}
\end{array}\right] .
$$


The vectors $c^{s}, z^{s}, c^{q}$, and $z^{q}$ are defined analogously. We will use corresponding notation for the search directions as well. Finally, let

$$
A^{T}=\left[\begin{array}{l}
A^{s} \\
A^{q} \\
A^{l}
\end{array}\right], x=\left[\begin{array}{l}
x^{s} \\
x^{q} \\
x^{l}
\end{array}\right], c=\left[\begin{array}{l}
c^{s} \\
c^{q} \\
c^{l}
\end{array}\right], z=\left[\begin{array}{l}
z^{s} \\
z^{q} \\
z^{l}
\end{array}\right],
$$

and

$$
n=\sum_{j=1}^{n_{s}} s_{j}+n_{q}+n_{l} .
$$

With the notations introduced above, the primal and dual equality constraints can be represented respectively as

$$
A x=b, \quad A^{T} y+z=c .
$$

In this paper, we assume that $A$ has full row rank. However, the preprocess option, when it is turned on, will correctly detect and remove dependent constraints.

The main step at each iteration of our algorithms is the computation of the search direction $(\Delta x, \Delta y, \Delta z)$ from the symmetrized Newton equation with respect to an invertible block diagonal scaling matrix $P$ for the semidefinite block and a block scaling matrix $G$ for the quadratic block. The matrices $P$ and $G$ are usually chosen as a function of the current iterate $x, z$ and we will elaborate on specific choices below. The search direction $(\Delta x, \Delta y, \Delta z)$ is obtained from the following system of equations:

$$
\begin{aligned}
& A^{T} \Delta y+\quad \Delta z=R_{d}:=c-z-A^{T} y \\
& A \Delta x \quad=r_{p}:=b-A x \\
& \mathcal{E}^{s} \Delta x^{s} \quad+\mathcal{F}^{s} \Delta z^{s}=R_{c}^{s}:=\operatorname{svec}\left(\sigma \mu I-H_{P}\left(\operatorname{smat}\left(x^{s}\right) \operatorname{smat}\left(z^{s}\right)\right)\right) \\
& \mathcal{E}^{q} \Delta x^{q} \quad+\mathcal{F}^{q} \Delta z^{q}=R_{c}^{q}:=\sigma \mu e^{q}-T_{G}\left(x^{q}, z^{q}\right) \\
& \mathcal{E}^{l} \Delta x^{l} \quad+\mathcal{F}^{l} \Delta z^{l}=R_{c}^{l}:=\sigma \mu e^{l}-\mathcal{E}^{l} \mathcal{F}^{l} e^{l},
\end{aligned}
$$

where $\mu=\langle x, z\rangle / n$ and $\sigma$ is the centering parameter. The notation smat denotes the inverse map of svec and both are to be interpreted as blockwise operators if the argument consists of blocks. Here $H_{P}$ is the symmetrization operator whose action on the $j$ th semidefinite block is defined by

$$
\begin{gathered}
H_{P_{j}}: \mathbb{R}^{s_{j} \times s_{j}} \longrightarrow \mathbb{R}^{s_{j} \times s_{j}} \\
H_{P_{j}}(U)=\frac{1}{2}\left[P_{j} U P_{j}^{-1}+P_{j}^{-T} U^{T} P_{j}^{T}\right],
\end{gathered}
$$

with $P_{j}$ the $j$ th block of the block diagonal matrix $P$ and $\mathcal{E}^{s}$ and $\mathcal{F}^{s}$ are symmetric block diagonal matrices whose $j$ th blocks are given by

$$
\mathcal{E}_{j}^{s}=P_{j} \circledast P_{j}^{-T} z_{j}^{s}, \quad \mathcal{F}_{j}^{s}=P_{j} x_{j}^{s} \circledast P_{j}^{-T},
$$


where $R \circledast T$ is the symmetrized Kronecker product operation described in [22].

In the quadratic block, $e^{q}$ denotes the blockwise identity vector, i.e.,

$$
e^{q}=\left[\begin{array}{c}
e_{1}^{q} \\
\vdots \\
e_{n_{q}}^{q}
\end{array}\right]
$$

where $e_{j}^{q}$ is the first unit vector in $\mathbb{R}^{q_{j}}$. Let the arrow operator defined in [3] be denoted by Arw (.). Thus Arw $(x)$ is a block diagonal matrix whose $i$ th block is

$$
\operatorname{Arw}\left(x^{i}\right):=\operatorname{Arw}\left(\begin{array}{c}
x_{0}^{i} \\
x_{1}^{i}
\end{array}\right):=\left(\begin{array}{cc}
x_{0}^{i} & x_{1}^{i T} \\
x_{1}^{i} & x_{0}^{i} I_{n_{i}}
\end{array}\right) .
$$

Then the operator $T_{G}\left(x^{q}, z^{q}\right)$ is defined as follows:

$$
T_{G}\left(x^{q}, z^{q}\right)=\left[\begin{array}{c}
\operatorname{Arw}\left(G_{1} x_{1}^{q}\right)\left(G_{1}^{-1} z_{1}^{q}\right) \\
\vdots \\
\operatorname{Arw}\left(G_{n_{q}} x_{n_{q}}^{q}\right)\left(G_{n_{q}}^{-1} z_{n_{q}}^{q}\right)
\end{array}\right],
$$

where $G$ is a symmetric block diagonal matrix that depends on $x, z$ and $G_{i}$ is the $i$ th block of $G$. The matrices $\mathcal{E}^{q}$ and $\mathcal{F}^{q}$ are block diagonal matrices whose the $i$ th blocks are given by

$$
\mathcal{E}_{i}^{q}=\operatorname{Arw}\left(G_{i}^{-1} z_{i}^{q}\right) G_{i}, \quad \mathcal{F}_{i}^{q}=\operatorname{Arw}\left(G_{i} x_{i}^{q}\right) G_{i}^{-1} .
$$

In the linear block, $e^{l}$ denotes the $n_{l}$-dimensional vector of ones, and $\mathcal{E}^{l}=$ $\operatorname{diag}\left(z^{l}\right), \mathcal{F}^{l}=\operatorname{diag}\left(x^{l}\right)$.

For future reference, we partition the vectors $R_{d}, \Delta x$, and $\Delta z$ in a manner analogous to $c, x$, and $z$ as follows:

$$
R_{d}=\left[\begin{array}{l}
R_{d}^{s} \\
R_{d}^{q} \\
R_{d}^{l}
\end{array}\right], \Delta x=\left[\begin{array}{c}
\Delta x^{s} \\
\Delta x^{q} \\
\Delta x^{l}
\end{array}\right], \Delta z=\left[\begin{array}{c}
\Delta z^{s} \\
\Delta z^{q} \\
\Delta z^{l}
\end{array}\right] .
$$

Assuming that $m=\mathcal{O}(n)$, we compute the search direction via a Schur complement equation as follows (the reader is referred to [2] and [22] for details). First compute $\Delta y$ from the Schur complement equation

$$
M \Delta y=h,
$$

where

$$
\begin{aligned}
M= & \left(A^{s}\right)^{T}\left(\mathcal{E}^{s}\right)^{-1} \mathcal{F}^{s} A^{s}+\left(A^{q}\right)^{T}\left(\mathcal{E}^{q}\right)^{-1} \mathcal{F}^{q} A^{q}+\left(A^{l}\right)^{T}\left(\mathcal{E}^{l}\right)^{-1} \mathcal{F}^{l} A^{l} \\
h= & r_{p}-\left(A^{s}\right)^{T}\left(\mathcal{E}^{s}\right)^{-1}\left(R_{c}^{s}-\mathcal{F}^{s} R_{d}^{s}\right) \\
& -\left(A^{q}\right)^{T}\left(\mathcal{E}^{q}\right)^{-1}\left(R_{c}^{q}-\mathcal{F}^{q} R_{d}^{q}\right)-\left(A^{l}\right)^{T}\left(\mathcal{E}^{l}\right)^{-1}\left(R_{c}^{l}-\mathcal{F}^{l} R_{d}^{l}\right) .
\end{aligned}
$$


Then compute $\Delta x$ and $\Delta z$ from the equations

$$
\begin{aligned}
\Delta z & =R_{d}-A^{T} \Delta y \\
\Delta x^{s} & =\left(\mathcal{E}^{s}\right)^{-1} R_{c}^{s}-\left(\mathcal{E}^{s}\right)^{-1} \mathcal{F}^{s} \Delta z^{s} \\
\Delta x^{q} & =\left(\mathcal{E}^{q}\right)^{-1} R_{c}^{q}-\left(\mathcal{E}^{q}\right)^{-1} \mathcal{F}^{q} \Delta z^{q} \\
\Delta x^{l} & =\left(\mathcal{E}^{l}\right)^{-1} R_{c}^{l}-\left(\mathcal{E}^{l}\right)^{-1} \mathcal{F}^{l} \Delta z^{l} .
\end{aligned}
$$

\subsection{Two choices of search directions}

We start by introducing some notation that we will use in the remainder of this paper. For a given $q_{i}$-dimensional vector $x_{i}^{q}$, we let $x_{i}^{0}$ denote its first component and $x_{i}^{1}$ denote its subvector consisting of the remaining entries, i.e.,

$$
\left[\begin{array}{c}
x_{i}^{0} \\
x_{i}^{1}
\end{array}\right]=\left[\begin{array}{c}
\left(x_{i}^{q}\right)_{1} \\
\left(x_{i}^{q}\right)_{2: q_{i}}
\end{array}\right] .
$$

We will use the same convention for $z_{i}^{q}, \Delta x_{i}^{q}$, etc. Also, we define the following function from $K_{q}^{q_{i}}$ to $\mathbb{R}_{+}$:

$$
\gamma\left(x_{i}^{q}\right):=\sqrt{\left(x_{i}^{0}\right)^{2}-\left\langle x_{i}^{1}, x_{i}^{1}\right\rangle} .
$$

Finally, we use $X$ and $Z$ for $\operatorname{smat}\left(x^{s}\right)$ and $\operatorname{smat}\left(z^{s}\right)$, where the operation is applied blockwise to form a block diagonal symmetric matrix of order $\sum_{j=1}^{n_{s}} s_{j}$.

In the current release of this package, the user has two choices of scaling operators parametrized by $P$ and $G$, resulting in two different search directions: the HKM direction $[10,12,16]$, and the NT direction [17]. See also Tsuchiya [26, 27 for the second-order case.

(1) The HKM direction. This choice uses the scaling matrix $P=Z^{1 / 2}$ for the semidefinite blocks and a symmetric block diagonal scaling matrix $G$ for the quadratic blocks where the $i$ th block $G_{i}$ is given by the following equation:

$$
G_{i}=\left[\begin{array}{cc}
z_{i}^{0} & \left(z_{i}^{1}\right)^{T} \\
z_{i}^{1} & \gamma\left(z_{i}^{q}\right) I+\frac{z_{i}^{1}\left(z_{i}^{1}\right)^{T}}{\gamma\left(z_{i}^{q}\right)+z_{i}^{0}}
\end{array}\right] .
$$

(2) The NT direction. This choice uses the scaling matrix $P=N^{-1}$ for the semidefinite blocks, where $N$ is a matrix such that $D:=N^{T} Z N=$ $N^{-1} X N^{-T}$ is a diagonal matrix [22], and $G$ is a symmetric block diagonal matrix whose $i$ th block $G_{i}$ is defined as follows. Let

$$
\omega_{i}=\sqrt{\frac{\gamma\left(z_{i}^{q}\right)}{\gamma\left(x_{i}^{q}\right)}}, \quad \xi_{i}=\left[\begin{array}{c}
\xi_{i}^{0} \\
\xi_{i}^{1}
\end{array}\right]=\left[\begin{array}{c}
\frac{1}{\omega_{i}} z_{i}^{0}+\omega_{i} x_{i}^{0} \\
\frac{1}{\omega_{i}} z_{i}^{1}-\omega_{i} x_{i}^{1}
\end{array}\right] .
$$


Then

$$
G_{i}=\omega_{i}\left[\begin{array}{cc}
t_{i}^{0} & \left(t_{i}^{1}\right)^{T} \\
t_{i}^{1} & I+\frac{t_{i}^{1}\left(t_{i}^{1}\right)^{T}}{1+t_{i}^{0}}
\end{array}\right], \quad \text { where }\left[\begin{array}{c}
t_{i}^{0} \\
t_{i}^{1}
\end{array}\right]=\frac{1}{\gamma\left(\xi_{i}\right)}\left[\begin{array}{c}
\xi_{i}^{0} \\
\xi_{i}^{1}
\end{array}\right]
$$

\subsection{Computation of the search directions}

The size and the density of the Schur complement matrix $M$ defined in (10) is the main determinant of the cost of each iteration in our algorithm. The density of this matrix depends on two factors: (i) The density of the constraint coefficient matrices $A^{s}, A^{q}$, and $A^{l}$, and (ii) any additional fill-in introduced because of the terms $\left(\mathcal{E}^{s}\right)^{-1} \mathcal{F}^{s},\left(\mathcal{E}^{q}\right)^{-1} \mathcal{F}^{q}$, and $\left(\mathcal{E}^{l}\right)^{-1} \mathcal{F}^{l}$ in $(10)$.

2.3.1. Semidefinite blocks For problems with semidefinite blocks, it appears that there is not much one can do about additional fill-in, since $\left(\mathcal{E}^{s}\right)^{-1} \mathcal{F}^{s}$ is dense and structure-less for most problems. One can take advantage of sparsity in $A^{s}$ in related computations, however, and we discussed some of these issues, such as blockwise computations, in our earlier papers $[22,25]$.

The way we exploit sparsity of $A_{j}^{s}$ in the computation of $M_{j}^{s}:=\left(A_{j}^{s}\right)^{T}\left(\mathcal{E}_{j}^{s}\right)^{-1} \mathcal{F}_{j}^{s} A_{j}^{s}$ basically follows the approach in [7]. We will not go into the details here but just briefly highlight one issue that is often critical in cutting down the computation time in forming $M_{j}^{s}$. Let $A_{j}^{s}(:, k)$ be the $k$ th column of $A_{j}^{s}$. In computing the $k$ th column of $M_{j}^{s}$, typically a matrix product of the form $x_{j}^{s} \operatorname{smat}\left(A_{j}^{s}(:, k)\right)\left(z_{j}^{s}\right)^{-1}$ or $w_{j}^{s} \operatorname{smat}\left(A_{j}^{s}(:, k)\right) w_{j}^{s}$ is required for the HKM direction or NT direction, respectively. In many large SDP problems, the matrix $\operatorname{smat}\left(A_{j}^{s}(:, k)\right)$ is usually very sparse, and it is important to store this matrix as a sparse matrix in MATLAB and perform sparse-dense matrix-matrix multiplication in the matrix products just mentioned whenever possible. Also, entries of this product only need to be computed if they contribute to an entry of $M$, i.e., if they correspond to a nonzero entry of $A_{j}^{s}\left(:, k^{\prime}\right)$ for some $k^{\prime}$.

2.3.2. Quadratic and linear blocks For linear blocks, $\left(\mathcal{E}^{l}\right)^{-1} \mathcal{F}^{l}$ is a diagonal matrix and it does not introduce any additional fill-in. This matrix does, however, affect the conditioning of the Schur complement matrix and is a popular subject of research in implementations of interior-point methods for linear programming.

From equation (11), it is easily shown that the contribution of the quadratic blocks to the matrix $M$ is given by

$$
M^{q}=\left(A^{q}\right)^{T}\left(\mathcal{E}^{q}\right)^{-1} \mathcal{F}^{q} A^{q}=\sum_{i=1}^{n_{q}} \underbrace{\left(A_{i}^{q}\right)^{T}\left(\mathcal{E}_{i}^{q}\right)^{-1} \mathcal{F}_{i}^{q} A_{i}^{q}}_{M_{i}^{q}} .
$$


For the HKM direction, $\left(\mathcal{E}^{q}\right)^{-1} \mathcal{F}^{q}$ is a block diagonal matrix whose $i$ th block is given by

$$
\begin{aligned}
& \left(\mathcal{E}_{i}^{q}\right)^{-1} \mathcal{F}_{i}^{q}=G_{i}^{-1} \operatorname{Arw}\left(G_{i} x_{i}^{q}\right) G_{i}^{-1} \\
& =\frac{1}{\gamma^{2}\left(z_{i}^{q}\right)}\left(\left\langle x_{i}^{q}, z_{i}^{q}\right\rangle\left[\begin{array}{cc}
-1 & 0 \\
0 & I
\end{array}\right]+\left[\begin{array}{c}
x_{i}^{0} \\
x_{i}^{1}
\end{array}\right]\left[\begin{array}{c}
z_{i}^{0} \\
-z_{i}^{1}
\end{array}\right]^{T}+\left[\begin{array}{c}
z_{i}^{0} \\
-z_{i}^{1}
\end{array}\right]\left[\begin{array}{c}
x_{i}^{0} \\
x_{i}^{1}
\end{array}\right]^{T}\right) .
\end{aligned}
$$

(Note that $\operatorname{Arw}\left(G_{i}^{-1} z_{i}^{q}\right)=I$.) Thus, we see that matrix $\left(\mathcal{E}_{i}^{q}\right)^{-1} \mathcal{F}_{i}^{q}$ in $M_{i}^{q}$ is the sum of a diagonal matrix and a rank-two symmetric matrix. Hence

$$
M_{i}^{q}=\frac{\left\langle x_{i}^{q}, z_{i}^{q}\right\rangle}{\gamma^{2}\left(z_{i}^{q}\right)}\left(A_{i}^{q}\right)^{T} J_{i} A_{i}^{q}+u_{i}^{q}\left(v_{i}^{q}\right)^{T}+v_{i}^{q}\left(u_{i}^{q}\right)^{T},
$$

where

$$
J_{i}=\left[\begin{array}{cc}
-1 & 0 \\
0 & I
\end{array}\right], \quad u_{i}^{q}=\left(A_{i}^{q}\right)^{T}\left[\begin{array}{l}
x_{i}^{0} \\
x_{i}^{1}
\end{array}\right], \quad v_{i}^{q}=\left(A_{i}^{q}\right)^{T}\left(\frac{1}{\gamma^{2}\left(z_{i}^{q}\right)}\left[\begin{array}{c}
z_{i}^{0} \\
-z_{i}^{1}
\end{array}\right]\right) .
$$

The appearance of the outer-product terms in the equation above is potentially alarming. If the vectors $u_{i}^{q}, v_{i}^{q}$ are dense, then even if $A_{i}^{q}$ is sparse, the corresponding matrix $M_{i}^{q}$, and hence the Schur complement matrix $M$, will be dense. A direct factorization of the resulting dense matrix will be very expensive for even moderately high $m$.

The observed behavior of the density of the Schur complement matrix on test problems depends largely on the particular problem structure. When the problem has many small quadratic blocks, it is often the case that each block appears in only a small fraction of the constraints. In this case, all $A_{i}^{q}$ matrices are sparse and the vectors $u_{i}^{q}$ and $v_{i}^{q}$ turn out to be sparse vectors for each $i$. Consequently, the Schur complement matrix remains relatively sparse for these problems and it can be factorized directly and cheaply. In Figure 1, the density structures of the Schur complement matrices in the first and later iterations of our algorithm applied to the the problem nq130 depict the situation and are typical for all nql and qssp problems. Since we initially choose multiples of unit vectors for our variables, all the nonzero elements of the Schur complement matrix in the first iteration come from the nonzero elements of the constraint matrices. Later iterations introduce fewer than $3 \%$ new nonzero elements.

The situation is drastically different for problems where one of the quadratic blocks, say the $i$ th block, is large. For such problems the vectors $u_{i}^{q}, v_{i}^{q}$ are typically dense, and therefore, $M_{i}^{q}$ is likely be a dense matrix even if the data $A_{i}^{q}$ is sparse. However, observe that $M_{i}^{q}$ is a rank-two perturbation of a sparse matrix when $A_{i}^{q}$ is sparse. In such a situation, it may be advantageous to use the Sherman-Morrison-Woodbury update formula [9] when solving the Schur complement equation (10). This is a standard strategy used in linear programming when there are dense columns in the constraint matrix and this is the approach we used in our implementation of SDPT3. This approach helps tremendously on the scheduling problems from the DIMACS Challenge set. Figure 2 depicts 

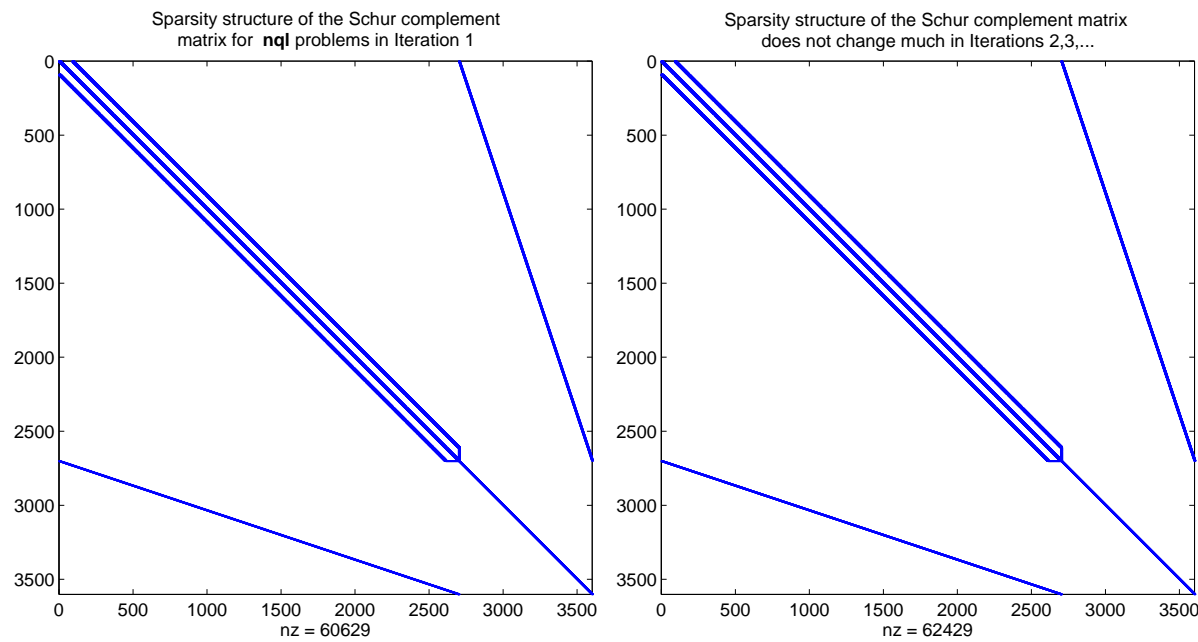

Fig. 1. The output of the spy function in MATLAB on the Schur complement matrix for nq130. Later iterations introduce less than $3 \%$ new nonzero elements.
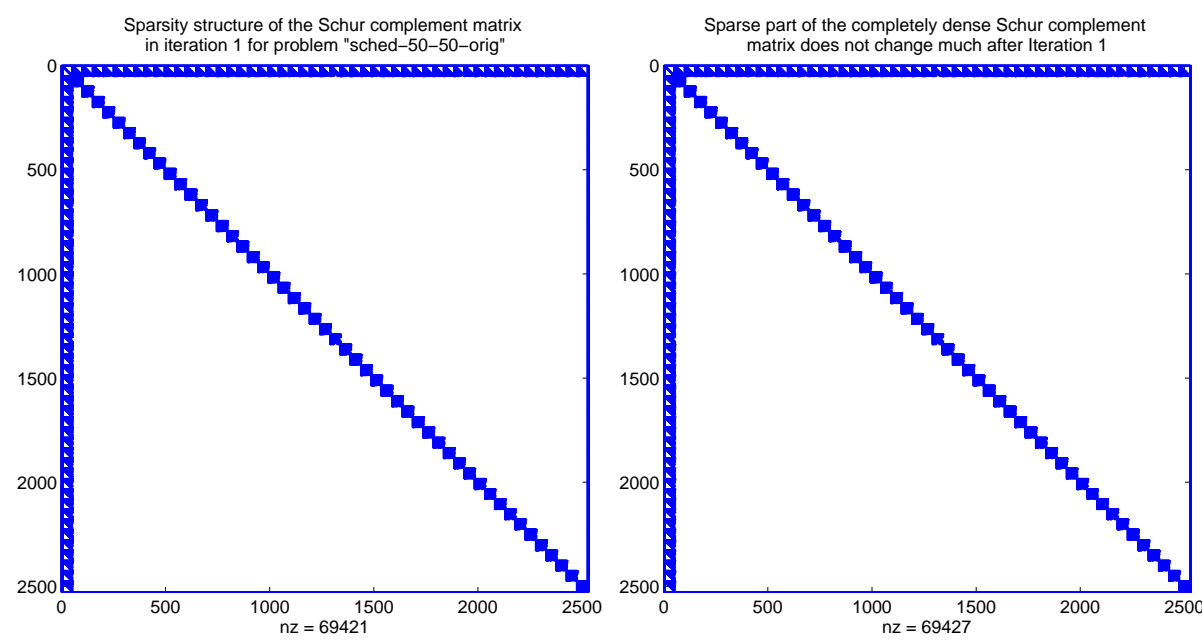

Fig. 2. The output of the spy function in MATLAB for problem sched-50-50-orig on (i) the complete Schur complement matrix in the first iteration, (ii) the sparse portion of the Schur complement matrix in the following iterations.

the Schur complement matrix $M$ in the first iteration and its sparse portion in the following iterations. While these two matrices have almost identical sparsity patterns, the complete Schur complement matrix becomes completely dense after the first iteration.

To apply the Sherman-Morrison-Woodbury formula, we need to modify the sparse portion of the matrix $M_{i}^{q}$ slightly. Since the diagonal matrix $J_{i}$ has a negative component, the matrix $\left(A_{i}^{q}\right)^{T} J_{i} A_{i}^{q}$ need not be a positive definite matrix, and therefore the Cholesky factorization of the sparse portion of $M_{i}^{q}$ need not 
exist. To overcome this problem, we use the following identity:

$$
M_{i}^{q}=\frac{\left\langle x_{i}^{q}, z_{i}^{q}\right\rangle}{\gamma^{2}\left(z_{i}^{q}\right)}\left(A_{i}^{q}\right)^{T} A_{i}^{q}+u_{i}^{q}\left(v_{i}^{q}\right)^{T}+v_{i}^{q}\left(u_{i}^{q}\right)^{T}-2 \frac{\left\langle x_{i}^{q}, z_{i}^{q}\right\rangle}{\gamma^{2}\left(z_{i}^{q}\right)} k_{i} k_{i}^{T},
$$

where $u_{i}^{q}$ and $v_{i}^{q}$ are as in (25) and

$$
k_{i}=\left(A_{i}^{q}\right)^{T} e_{i}^{q}
$$

Note that if $A_{i}^{q}$ is a large sparse matrix with a few dense rows, we also use the Sherman-Morrison-Woodbury formula to handle the matrix $\left(A_{i}^{q}\right)^{T} A_{i}^{q}$ in (26).

We end our discussion on the computation of the HKM direction with the following formula that is needed in the computation of the right-hand-side vector (12):

$$
\left(\mathcal{E}_{i}^{q}\right)^{-1}\left(R_{c}^{q}\right)_{i}=\frac{\sigma \mu}{\gamma^{2}\left(z_{i}^{q}\right)}\left[\begin{array}{c}
z_{i}^{0} \\
-z_{i}^{1}
\end{array}\right]-x_{i}^{q}
$$

Just as for the HKM direction, we can obtain a very simple formula for $\left(\mathcal{E}_{i}^{q}\right)^{-1} \mathcal{F}_{i}^{q}$ for the NT direction. By noting that $G_{i} x_{i}^{q}=G_{i}^{-1} z_{i}^{q}$, it is easy to see that the $i$ th block $\left(\mathcal{E}_{i}^{q}\right)^{-1} \mathcal{F}_{i}^{q}=G_{i}^{-2}$, and a rather straightforward algebraic manipulation gives the following identity:

$$
\left(\mathcal{E}_{i}^{q}\right)^{-1} \mathcal{F}_{i}^{q}=G_{i}^{-2}=\frac{1}{\omega_{i}^{2}}\left(\left[\begin{array}{cc}
-1 & 0 \\
0 & I
\end{array}\right]+2\left[\begin{array}{c}
t_{i}^{0} \\
-t_{i}^{1}
\end{array}\right]\left[\begin{array}{c}
t_{i}^{0} \\
-t_{i}^{1}
\end{array}\right]^{T}\right)
$$

For the NT direction, the formula in (28) also holds and we have:

$$
M_{i}^{q}=\frac{1}{\omega_{i}^{2}}\left(\left(A_{i}^{q}\right)^{T} J_{i} A_{i}^{q}+2 u_{i}^{q}\left(u_{i}^{q}\right)^{T}\right), \text { with } u_{i}^{q}=\left(A_{i}^{q}\right)^{T}\left[\begin{array}{c}
t_{i}^{0} \\
-t_{i}^{1}
\end{array}\right]
$$

We note that the identity (30) describing the NT direction was observed by other authors - see, e.g., [8]. The identities (23) and (24), however, appear to be new in the literature. It is straightforward, if a bit tedious, to verify these formulas. In addition to simplifying the search direction computation, these identities can be used to provide a simple proof of the scale-invariance of the HKM search direction in second-order cone programming. In [27], Tsuchiya proves this result and the scale-invariance of the NT direction using two-page arguments for each proof. We refer the reader to [22] for a description of scale-invariance and provide the following simple and instructive proof:

Proposition 1. Consider a pure second-order cone programming problem $\left(n_{s}=\right.$ 0 and $\left.n_{l}=0\right)$. The HKM and NT directions for this problem are scale-invariant. 
Proof. The scaled problem is constructed as follows: Let $F_{i} \in \mathcal{G}_{i}$ denote a scaling matrix for block $i$ where $\mathcal{G}_{i}$ is the automorphism group of the cone $K_{q}^{q_{i}}$. For future reference, note that we have

$$
F_{i}^{T} \bar{J}_{i} F_{i}=\bar{J}_{i}, \quad F_{i}^{T} \bar{J}_{i}=\bar{J}_{i} F_{i}^{-1}, \quad \bar{J}_{i} F_{i}=F_{i}^{-T} \bar{J}_{i}, \quad \bar{J}_{i} F_{i}^{T}=F_{i}^{-1} \bar{J}_{i},
$$

where $\bar{J}_{i}=-J_{i}$, and $J_{i}$ is as in $(25)$. Let $F=\operatorname{diag}\left[F_{1}, \ldots, F_{n_{q}}\right]$ and define the scaled quantities as follows:

$$
\hat{A}^{q}=F^{-1} A^{q}, \quad \hat{b}=b, \quad \hat{c}^{q}=F^{-1} c^{q}, \quad \hat{x}^{q}=F^{T} x^{q}, \quad \hat{y}=y, \quad \hat{z}^{q}=F^{-1} z^{q} .
$$

Note that $\hat{r}_{p}=r_{p}$ and $\hat{R}_{d}=F^{-1} R_{d}$. First, we consider the HKM direction. We observe that $\gamma^{2}\left(\hat{z}_{i}^{q}\right)=\left(\hat{z}_{i}^{q}\right)^{T} \bar{J}_{i} \hat{z}_{i}^{q}=\left(z_{i}^{q}\right)^{T} F_{i}^{-T} \bar{J}_{i} F_{i}^{-1} z_{i}^{q}=\gamma^{2}\left(z_{i}^{q}\right)$. Now, from equation (24) and (31) it follows that each $M_{i}^{q}$, and therefore $M^{q}$, is invariant with respect to this automorphic scaling. Using (28), we see that $h$ in (12) is also invariant. Now, if we denote the HKM search direction for the scaled problem by $\left(\widehat{\Delta x}^{q}, \widehat{\Delta y}, \widehat{\Delta z}^{q}\right)$ and the corresponding direction for the unscaled problem by $\left(\Delta x^{q}, \Delta y, \Delta z^{q}\right)$ we immediately obtain $\widehat{\Delta y}=\Delta y$ from equation (10), $\widehat{\Delta z}^{q}=F^{-1} \Delta z^{q}$ from (13) and $\widehat{\Delta x}^{q}=F^{T} \Delta x^{q}$ from (15) and (28). Thus, the HKM direction for SOCP is scale-invariant.

To prove the result for the NT direction, we first observe that $\gamma\left(\hat{x}_{i}^{q}\right)=\gamma\left(x_{i}^{q}\right)$ and that $\omega_{i}$ defined in (20) remains unchanged after scaling. The scaled equivalent of $\xi_{i}$ defined in $(20)$ is $\hat{\xi}_{i}=\frac{1}{\omega_{i}} \hat{z}_{i}^{q}+\omega_{i} \bar{J}_{i} \hat{x}_{i}^{q}=F_{i}^{-1}\left(\frac{1}{\omega_{i}} z_{i}^{q}+\omega_{i} \bar{J}_{i} x_{i}^{q}\right)=F_{i}^{-1} \xi_{i}$. Thus, with the scaled quantities, we obtain

$$
\hat{t}_{i}=\left[\begin{array}{c}
\hat{t}_{i}^{0} \\
\hat{t}_{i}^{1}
\end{array}\right]=F_{i}^{-1}\left[\begin{array}{c}
t_{i}^{0} \\
t_{i}^{1}
\end{array}\right]
$$

Now, from equation (30) and (31) it follows that each $M_{i}^{q}$, and therefore $M^{q}$, is invariant with respect to this automorphic scaling. Continuing as above, we conclude that the NT direction for SOCP must be scale-invariant as well.

\subsection{Step-length computation}

Once a direction $\Delta x$ is computed, a full step will not be allowed if $x+\Delta x$ violates the conic constraints. Thus, the next iterate must take the form $x+\alpha \Delta x$ for an appropriate choice of the step-length $\alpha$. In this subsection, we discuss an efficient strategy to compute the step-length $\alpha$.

For semidefinite blocks, it is straightforward to verify that, for the $j$ th block, the maximum allowed step-length that can be taken without violating the positive semidefiniteness of the matrix $x_{j}^{s}+\alpha_{j}^{s} \Delta x_{j}^{s}$ is given as follows:

$$
\alpha_{j}^{s}= \begin{cases}\frac{-1}{\lambda_{\min }\left(\left(x_{j}^{s}\right)^{-1} \Delta x_{j}^{s}\right)}, & \text { if the minimum eigenvalue } \lambda_{\min } \text { is negative } \\ \infty & \text { otherwise. }\end{cases}
$$


If the computation of eigenvalues necessary in $\alpha_{j}^{s}$ above becomes expensive, then we resort to finding an approximation of $\alpha_{j}^{s}$ by estimating extreme eigenvalues using Lanczos iterations [24]. This approach is quite accurate in general and represents a good trade-off between the effort versus quality of the resulting stepsizes.

For quadratic blocks, the largest step-length $\alpha_{i}^{q}$ that keeps the next iterate feasible with respect to the $k$ th quadratic cone can be computed as follows. Let

$$
a_{i}=\gamma^{2}\left(\Delta x_{i}^{q}\right), \quad b_{i}=\left\langle\Delta x_{i}^{q},-J_{i} x_{i}^{q}\right\rangle, \quad c_{i}=\gamma^{2}\left(x_{i}^{q}\right)
$$

where $J_{i}$ is the matrix defined in $(25)$ and let

$$
d_{i}=b_{i}^{2}-a_{i} c_{i}
$$

We want the largest $\alpha$ with $a_{i} \alpha^{2}+2 b_{i} \alpha+c_{i}>0$ for all smaller positive values. This is given by

$$
\alpha_{i}^{q}= \begin{cases}\frac{-b_{i}-\sqrt{d_{i}}}{a_{i}} & \text { if } a_{i}<0 \text { or } b_{i}<0, a_{i} \leq b_{i}^{2} / c_{i} \\ \frac{-c_{i}}{2 b_{i}} & \text { if } a_{i}=0, b_{i}<0 \\ \infty & \text { otherwise. }\end{cases}
$$

For the linear block, the maximum allowed step-length $\alpha_{i}^{l}$ for the $h$ th component is given by

$$
\alpha_{h}^{l}= \begin{cases}\frac{-x_{h}^{l}}{\Delta x_{h}^{l}}, & \text { if } \Delta x_{h}^{l}<0 \\ \infty & \text { otherwise }\end{cases}
$$

Finally, an appropriate step-length $\alpha$ that can be taken in order for $x+\alpha \Delta x$ to satisfy all the conic constraints takes the form

$$
\alpha=\min \left(1, \gamma \min _{1 \leq j \leq n_{s}} \alpha_{j}^{s}, \gamma \min _{1 \leq i \leq n_{q}} \alpha_{i}^{q}, \gamma \min _{1 \leq h \leq n_{l}} \alpha_{h}^{l}\right)
$$

where $\gamma$ (known as the step-length parameter) is typically chosen to be a number slightly less than 1, for example as in the adaptive scheme shown in Algorithm IPC, to ensure that the next iterate $x+\alpha \Delta x$ stays strictly in the interior of all the cones.

For the dual direction $\Delta z$, we let the analog of $\alpha_{j}^{s}, \alpha_{i}^{q}$ and $\alpha_{h}^{l}$ be $\beta_{j}^{s}, \beta_{i}^{q}$ and $\beta_{h}^{l}$, respectively. Similar to the primal direction, the step-length that can be taken by the dual direction $\Delta z$ is given by

$$
\beta=\min \left(1, \gamma \min _{1 \leq j \leq n_{s}} \beta_{j}^{s}, \gamma \min _{1 \leq i \leq n_{q}} \beta_{i}^{q}, \gamma \min _{1 \leq h \leq n_{l}} \beta_{h}^{l}\right) .
$$




\subsection{Sherman-Morrison-Woodbury formula and iterative refinement}

In this subsection, we discuss how we solve the Schur complement equation when $M$ is a low rank perturbation of a sparse matrix. As discussed in Section 2.3 such situations arise when the SQLP does not have a semidefinite block, but has large quadratic blocks or the constraint matrices $A_{i}^{q}, A^{l}$ have a small number of dense rows. In such a case, the Schur complement matrix $M$ can be written in the form

$$
M=H+U V^{T}
$$

where $H$ is a sparse symmetric matrix and $U, V$ have only few columns. If $H$ is non-singular, then by the Sherman-Morrison-Woodbury formula, the solution of the Schur complement equation is given by

$$
\Delta y=\hat{h}-H^{-1} U\left(I+V^{T} H^{-1} U\right)^{-1} V^{T} \hat{h},
$$

where $\hat{h}=H^{-1} h$.

Computing $\Delta y$ via the Sherman-Morrison-Woodbury update formula above is not always stable, and the computed solution for $\Delta y$ can be highly inaccurate when $H$ is ill-conditioned. To overcome such a difficulty, we combine the Sherman-Morrison-Woodbury update with iterative refinement [11]. It is noted in [11] that iterative refinement is beneficial even if the residuals are computed only at the working precision. Our numerical experience with the SQLP problems from the DIMACS Challenge set confirmed that iterative refinement very often does greatly improve the accuracy of the computed solution for $\Delta y$ via the Sherman-Morrison-Woodbury formula. However, we must mention that iterative refinement can occasionally fail to provide any significant improvement. We have not yet incorporated a stable and efficient method for computing $\Delta y$ when $M$ has the form (35), but note that Goldfarb and Scheinberg [8] discuss a stable product-form Cholesky factorization approach to this problem.

\section{Initial iterates}

Our algorithms can start with an infeasible starting point. However, the performance of these algorithms is quite sensitive to the choice of the initial iterate. As observed in [7], it is desirable to choose an initial iterate that at least has the same order of magnitude as an optimal solution of the SQLP. If a feasible starting point is not known, we recommend that the following initial iterate be used:

$$
\begin{gathered}
y^{0}=0, \\
\left(x_{j}^{s}\right)^{0}=\xi_{j}^{s} I_{s_{j}}, \quad\left(z_{j}^{s}\right)^{0}=\eta_{j}^{s} I_{s_{j}}, \quad j=1, \ldots, n_{s}, \\
\left(x_{i}^{q}\right)^{0}=\xi_{i}^{q} e_{i}^{q}, \quad\left(z_{i}^{q}\right)^{0}=\eta_{i}^{q} e_{i}^{q}, \quad i=1, \ldots, n_{q}, \\
\left(x^{l}\right)^{0}=\xi^{l} e^{l}, \quad\left(z^{l}\right)^{0}=\eta^{l} e^{l},
\end{gathered}
$$


where $I_{s_{j}}$ is the identity matrix of order $s_{j}$, and

$$
\begin{gathered}
\xi_{j}^{s}=s_{j} \max _{1 \leq k \leq m} \frac{1+\left|b_{k}\right|}{1+\left\|A_{j}^{s}(:, k)\right\|}, \quad \eta_{j}^{s}=\frac{1}{\sqrt{s_{j}}}\left[1+\max _{k}\left(\max _{k}\left\{\left\|A_{j}^{s}(:, k)\right\|\right\},\left\|c_{j}^{s}\right\|_{F}\right)\right], \\
\xi_{i}^{q}=\sqrt{q_{i}} \max _{1 \leq k \leq m} \frac{1+\left|b_{k}\right|}{1+\left\|A_{i}^{q}(:, k)\right\|}, \quad \eta_{i}^{q}=\sqrt{q_{i}}\left[1+\max \left(\max _{k}\left\{\left\|A_{i}^{q}(:, k)\right\|\right\},\left\|c_{i}^{q}\right\|\right)\right], \\
\xi^{l}=\max _{1 \leq k \leq m} \frac{1+\left|b_{k}\right|}{1+\left\|A^{l}(:, k)\right\|}, \quad \eta^{l}=1+\max \left(\max _{k}\left\{\left\|A^{l}(:, k)\right\|\right\},\left\|c^{l}\right\|\right),
\end{gathered}
$$

where $A_{j}^{s}(:, k)$ denotes the $k$ th column of $A_{j}^{s}$, and $A_{i}^{q}(:, k)$ and $A_{i}^{l}(:, k)$ are defined similarly.

By multiplying the identity matrix $I_{s_{i}}$ by the factors $\xi_{i}^{s}$ and $\eta_{i}^{s}$ for the semidefinite blocks, and similarly for the quadratic and linear blocks, the initial iterate has a better chance of having the appropriate order of magnitude.

The above iterate is the default in SDPT3, but other options are also available.

\section{Some implementation details}

SDPT3 version 3.0 (henceforth denoted SDPT3-3.0) implements the infeasible path-following algorithms described in Section 2. It is designed to be fairly flexible in the strategies used, allowing either the HKM or the NT search direction, switching on or off scaling of the problem and/or the predictor-corrector scheme, giving a choice of step-length determination, etc. (However, as we describe in the next section, the still available version 2.3 (denoted SDPT3-2.3) is more flexible, allowing two more search directions as well as homogeneous self-dual algorithms: we discuss below why these possibilities have been removed from the current version.) Details of these options can be found in the user's guide [28], available from the web sites named in the introduction. The computational results given in the next section were all obtained with default settings, except that we tested both search directions.

The output of SDPT3 is also flexible. Generally the output variables (X,y, Z) provide approximately optimal solutions, but if the output variable info(1) is 1 the problem is suspected to be primal infeasible and $(y, z)$ is an approximate certificate of infeasibility, with $\mathrm{b}^{T} \mathrm{y}=1, \mathrm{Z}$ in the appropriate cone, and $\mathrm{A}^{T} \mathrm{y}+\mathrm{Z}$ small, while if info(1) is 2 the problem is suspected to be dual infeasible and $\mathrm{X}$ is an approximate certificate of infeasibility, with $\langle\mathrm{C}, \mathrm{X}\rangle=-1, \mathrm{X}$ in the appropriate cone, and A X small. In the case that an indication of infeasibility is given, the final iterates are still available to the user.

\section{Mex files used.}

Our software uses a number of Mex routines generated from $\mathrm{C}$ programs written to carry out certain operations for which MATLAB is not efficient. In particular, operations such as extracting selected elements of a matrix, and performing 
arithmetic operations on these selected elements, are all done in C. As an example, the vectorization operation svec is coded in the $\mathrm{C}$ program mexsvec.c.

We also use a number of Mex routines generated from the Fortran programs for sparse Cholesky factorization discussed in Section 5.1.

\section{Cell array representation for problem data.}

Our implementation SDPT3 exploits the block structure of the given SQLP problem. In the internal representation of the problem data, we classify each semidefinite block into one of the following two types:

1. a dense or sparse matrix of dimension greater than or equal to 30 ;

2. a sparse block-diagonal matrix consisting of numerous sub-blocks each of dimension less than 30 .

The reason for using the sparse matrix representation to handle the case when we have numerous small diagonal blocks is that it is less efficient for MATLAB to work with a large number of cell array elements compared to working with a single cell array element consisting of a large sparse block-diagonal matrix. Technically, no problem will arise if one chooses to store the small blocks individually instead of grouping them together as a sparse block-diagonal matrix.

For the quadratic part, we typically group all quadratic blocks (small or large) into a single block, though it is not mandatory to do so. If there are a large number of small blocks, it is advisable to group them all together as a single large block consisting of numerous small sub-blocks for the same reason we mentioned before.

Let $L=n_{s}+n_{q}+1$. For each SQLP problem, the block structure of the problem data is described by an $L \times 2$ cell array named blk, The content of each of the elements of the cell arrays is given as follows. If the $j$ th block is a semidefinite block consisting of a single block of size $\mathbf{s}_{j}$, then

$$
\begin{aligned}
& \operatorname{blk}\{j, 1\}=' s ' \quad \text { blk }\{j, 2\}=\left[s_{j}\right] \\
& A\{j\}=\left[\bar{s}_{j} \times m \text { sparse }\right] \\
& C\{j\}, X\{j\}, Z\{j\}=\left[s_{j} \times s_{j} \text { double or sparse }\right],
\end{aligned}
$$

where $\bar{s}_{j}=s_{j}\left(s_{j}+1\right) / 2$.

If the $j$ th block is a semidefinite block consisting of numerous small subblocks, say $p$ of them, of dimensions $\mathbf{s}_{\mathrm{j} 1}, \mathbf{s}_{\mathrm{j} 2}, \ldots, \mathbf{s}_{\mathrm{jp}}$ such that $\sum_{k=1}^{p} \mathbf{s}_{\mathrm{jk}}=\mathbf{s}_{\mathrm{j}}$, then

$$
\begin{aligned}
& \operatorname{blk}\{j, 1\}=' s ' \quad \operatorname{blk}\{j, 2\}=\left[s_{j 1} s_{j 2} \cdots s_{j p}\right] \\
& A\{j\}=\left[\bar{s}_{j} \times m \text { sparse }\right] \\
& C\{j\}, X\{j\}, Z\{j\}=\left[s_{j} \times s_{j} \text { sparse }\right],
\end{aligned}
$$

where $\overline{\mathbf{s}}_{j}=\sum_{\mathrm{k}=1}^{\mathrm{p}} \mathrm{s}_{\mathrm{jk}}\left(\mathrm{s}_{\mathrm{jk}}+1\right) / 2$.

The above storage scheme for the data matrix $A_{j}^{s}$ associated with the semidefinite blocks of the SQLP problem represents a departure from earlier versions of our implementation, such as the one described in [25] and SDPT3-2.3. Previously, 
the semidefinite part of A was represented by an $n_{s} \times m$ cell array, where $\mathrm{A}\{\mathrm{j}, \mathrm{k}\}$ corresponds to the $k$ th constraint matrix associated with the $j$ th semidefinite block, and it was stored as an individual matrix in either dense or sparse format. Now, we store all the constraint matrices associated with the $j$ th semidefinite block in vectorized form as a single $\bar{s}_{j} \times m$ matrix where the $k$ th column of this matrix corresponds to the $k$ th constraint matrix. The data format we used in earlier versions of SDPT3 was more natural but, for the sake of computational efficiency, we adopted our current data representation. The reason for such a change is again due to the fact that it is less efficient for MATLAB to work with a single cell array with many cells. We also avoid explicit loops over the index $k$. In the next section, we will discuss the consequence of this modification in our storage scheme.

If the $i$ th block is a quadratic block consisting of numerous sub-blocks, say $p$ of them, of dimensions $\mathrm{q}_{\mathrm{i} 1}, \mathrm{q}_{\mathrm{i} 2}, \ldots, \mathrm{q}_{\mathrm{ip}}$ such that $\sum_{k=1}^{p} \mathrm{q}_{\mathrm{ik}}=\mathrm{q}_{\mathrm{i}}$, then

$$
\begin{aligned}
& \operatorname{blk}\{i, 1\}=' q ' \quad \operatorname{blk}\{i, 2\}=\left[\begin{array}{llll}
q_{i 1} & q_{i 2} & \cdots & q_{i p}
\end{array}\right] \\
& A\{i\}=\left[q_{i} \times m \text { sparse }\right] \\
& C\{i\}, X\{i\}, Z\{i\}=\left[q_{i} \times 1 \quad \text { double or sparse }\right] .
\end{aligned}
$$

If the $i$ th block is the linear block, then

$$
\begin{aligned}
& \operatorname{blk}\{i, 1\}=' 1, \quad \operatorname{blk}\{i, 2\}=n_{1} \\
& A\{i\}=\left[n_{1} \times m \text { sparse }\right] \\
& C\{i\}, X\{i\}, Z\{i\}=\left[n_{1} \times 1 \text { double or sparse }\right] .
\end{aligned}
$$

\section{Caveats.}

We should mention that "solving" SQLPs is more subtle than linear programming. For example, it is possible that both primal and dual problems are feasible, but their optimal values are not equal. Also, either problem may be infeasible without there being a certificate of that fact (so-called weak infeasibility). In such cases, our software package is likely to terminate after some iterations with an indication of short step-length or lack of progress. Also, even if there is a certificate of infeasibility, our infeasible-interior-point methods may not find it. (However, in our limited testing on randomly generated strongly infeasible problems, our algorithms have been quite successful in detecting infeasibility.)

\section{Computational experiments}

Here we describe the results of our computational testing of SDPT3, on problems from the SDPLIB collection of Borchers [4] as well as the DIMACS Challenge test problems [19]. In both, we solve a selection of the problems; in the DIMACS problems, these are selected as the more tractable problems, while our subset of the SDPLIB problems is more representative (but we cannot solve the largest two maxG problems). Since our algorithm is a primal-dual method storing the 


\begin{tabular}{|c|c|c|c|c|c|}
\hline Problem & $\mathrm{m}$ & $\mathrm{SD}$ & $\mathrm{SO}$ & $\mathrm{L}$ & opt. obj. value \\
\hline $\mathrm{bm} 1$ & 883 & 882 & (- & - - & 23.43982 \\
\hline copo14 & 1275 & {$[14 \times 14]$} & - & 364 & 0 \\
\hline copo23 & 5820 & {$[23 \times 23]$} & - & 1771 & 0 \\
\hline filter48-socp & 969 & 48 & 49 & 931 & 1.41612901 \\
\hline filtinf1 & 983 & 49 & 49 & 945 & primal inf. \\
\hline hamming-7-5-6 & 1793 & 128 & - & - & $42 \frac{2}{3}$ \\
\hline hamming-9-8 & 2305 & 512 & - & - & 224 \\
\hline hinf12 & 43 & 24 & - & - & $-0.0231(?)$ \\
\hline hinf13 & 57 & 30 & - & - & $-44.38(?)$ \\
\hline minphase & 48 & 48 & - & - & 5.98 \\
\hline $\mathrm{nb}$ & 123 & - & {$[793 \times 3]$} & 4 & -0.05070309 \\
\hline nb-L1 & 915 & - & {$[793 \times 3]$} & 797 & -13.01227 \\
\hline nb-L2 & 123 & - & {$[1637,838 \times 3]$} & 4 & -1.628972 \\
\hline nb-L2-bessel & 123 & - & {$[123,838 \times 3]$} & 4 & -0.102571 \\
\hline nql30 & 3680 & - & {$[900 \times 3]$} & 3602 & -0.9460 \\
\hline nql60 & 14560 & - & {$[3600 \times 3]$} & 14402 & -0.935423 \\
\hline nql180 & 130080 & - & {$[32400 \times 3]$} & 129602 & -0.927717 \\
\hline nql30old & 3601 & - & {$[900 \times 3]$} & 5560 & -0.9460 \\
\hline nql60old & 14401 & - & {$[3600 \times 3]$} & 21920 & -0.935423 \\
\hline nql180old & 129601 & - & {$[32400 \times 3]$} & 195360 & -0.927717 \\
\hline qssp30 & 3691 & - & {$[1891 \times 4]$} & 2 & -6.496675 \\
\hline qssp60 & 14581 & - & {$[7381 \times 4]$} & 2 & -6.562696 \\
\hline qssp180 & 130141 & - & {$[65341 \times 4]$} & 2 & -6.639527 \\
\hline qssp30old & 5674 & - & {$[1891 \times 4]$} & 3600 & -6.496675 \\
\hline qssp60old & 22144 & - & {$[7381 \times 4]$} & 14400 & -6.562696 \\
\hline qssp180old & 196024 & - & {$[65341 \times 4]$} & 129600 & -6.639527 \\
\hline sched-50-50-orig & 2527 & - & {$[2474,3]$} & 2502 & 26,673 \\
\hline sched-50-50-scaled & 2526 & - & 2475 & 2502 & 7.852038 \\
\hline sched-100-50-orig & 4844 & - & {$[4741,3]$} & 5002 & 181,889 \\
\hline sched-100-50-scaled & 4843 & - & 4742 & 5002 & 67.166281 \\
\hline sched-100-100-orig & 8338 & - & {$[8235,3]$} & 10002 & 717,367 \\
\hline sched-100-100-scaled & 8337 & - & 8236 & 10002 & 27.331457 \\
\hline sched-200-100-orig & 18087 & - & {$[17884,3]$} & 20002 & 141,360 \\
\hline sched-200-100-scaled & 18086 & - & 17885 & 20002 & 51.812471 \\
\hline torusg3-8 & 512 & 512 & - & - & 457.358179 \\
\hline toruspm3-8-50 & 512 & 512 & - & - & 527.808663 \\
\hline truss 5 & 208 & {$[33 \times 10,1]$} & - & - & 132.6356779 \\
\hline truss 8 & 496 & {$[33 \times 19,1]$} & - & - & 133.1145891 \\
\hline
\end{tabular}

Table 1. Selected DIMACS Challenge Problems. SD, SO, and L stand for semidefinite, secondorder, and linear blocks, respectively. Notation like [33 x 19] indicates that there were 33 semidefinite blocks, each a symmetric matrix of order 19 , etc.

primal iterate $\mathrm{X}$, it cannot exploit common sparsity in $\mathrm{C}$ and the constraint matrix as well as dual methods or nonlinear-programming based methods. We are therefore unable to solve the largest problems.

All results given below were obtained on a Pentium III PC $(800 \mathrm{MHz})$ with $1 \mathrm{G}$ of memory running Linux, using MATLAB 6.0. The test problems are listed in Tables 1 and 2, along with their dimensions. We also list optimal objective values of these problems that are reported in [19] and [4]. 


\begin{tabular}{|c|c|c|c|c|}
\hline Problem & $\mathrm{m}$ & semidefinite blocks & linear block & opt. obj. value \\
\hline arch8 & $\overline{174}$ & 161 & $\overline{174}$ & $\overline{7.05698}$ \\
\hline control7 & 666 & {$[70,35]$} & - & 20.6251 \\
\hline control10 & 1326 & {$[100,50]$} & - & 38.533 \\
\hline control11 & 1596 & {$[110,55]$} & - & 31.959 \\
\hline gpp250-4 & 251 & 250 & - & -747.3 \\
\hline gpp500-4 & 501 & 500 & - & -1567.02 \\
\hline hinf15 & 91 & 37 & - & 25 \\
\hline mcp250-1 & 250 & 250 & - & 317.2643 \\
\hline mcp500-1 & 500 & 500 & - & 598.1485 \\
\hline qap9 & 748 & 82 & - & $-1410^{\dagger}$ \\
\hline qap10 & 1021 & 101 & - & $-1093^{\dagger}$ \\
\hline ss30 & 132 & 294 & 132 & 20.2395 \\
\hline theta3 & 1106 & 150 & - & 42.16698 \\
\hline theta 4 & 1949 & 200 & - & 50.32122 \\
\hline theta 5 & 3028 & 250 & - & 57.23231 \\
\hline theta 6 & 4375 & 300 & - & 63.47709 \\
\hline truss7 & 86 & {$[150 \times 2,1]$} & - & -90.0001 \\
\hline truss 8 & 496 & {$[33 \times 19,1]$} & - & -133.1146 \\
\hline equalG11 & 801 & 801 & - & 629.1553 \\
\hline equalG51 & 1001 & 1001 & - & 4005.601 \\
\hline equalG32 & 2001 & 2001 & - & $\mathrm{N} / \mathrm{A}$ \\
\hline $\max G 11$ & 800 & 800 & - & 629.1648 \\
\hline $\max G 51$ & 1000 & 1000 & - & $4003.809^{\dagger}$ \\
\hline $\operatorname{maxG} 32$ & 2000 & 2000 & - & 1567.640 \\
\hline qpG11 & 800 & 1600 & - & 2448.659 \\
\hline qpG112 & 800 & 800 & 800 & 2448.659 \\
\hline qpG51 & 1000 & 2000 & - & $1181.000^{\dagger}$ \\
\hline qpG512 & 1000 & 1000 & 1000 & $1181.000^{\dagger}$ \\
\hline thetaG11 & 2401 & 801 & - & 400.00 \\
\hline thetaG11n & 1600 & 800 & - & 400.00 \\
\hline thetaG51 & 6910 & 1001 & - & 349.00 \\
\hline thetaG51n & 5910 & 1000 & - & 349.00 \\
\hline
\end{tabular}

Table 2. Selected SDPLIB Problems. Note that qpG112 is identical to qpG11 except that the structure of the semidefinite block is exposed as a sparse symmetric matrix of order 800 and a diagonal block of the same order, which can be viewed as a linear block, and similarly for qpG512. Also, thetaG11n is a more compact formulation of thetaG11, and similarly for thetaG51n.

$\dagger$ For some problems, we obtained the following alternative objective values that we believe to be more accurate: qap9: -1409.8, qap10: -1092.4, equalG32: 1567.627, maxG51: 4006.256, qpG51: 1181.800 .

\subsection{Cholesky factorization}

Earlier versions of SDPT3 were intended for problems that always have semidefinite cone constraints. As we indicated above, for such problems, the Schur complement matrix $M$ in (11) is a dense matrix after the first iteration. To solve the associated linear system (10), we first find a Cholesky factorization of $M$ and then solve two triangular systems. When $M$ is dense, a reordering of the rows and columns of $M$ does not alter the efficiency of the Cholesky factorization and specialized sparse Cholesky factorization routines are not useful. Therefore, earlier versions of SDPT3 (up to version 1.3) simply used MATLAB's chol routine for Cholesky factorizations. For versions 2.1 and 2.2, we introduced our own Cholesky factorization routine mexchol that utilizes loop unrolling and provided 
2-fold speed-ups on some architectures compared to MATLAB's chol routine. However, in newer versions of MATLAB that use numerics libraries based on LAPACK, MATLAB's chol routine is more efficient than our Cholesky factorization routine mexchol for dense matrices. Thus, in SDPT3-3.0, we use MATLAB's chol routine whenever $M$ is dense. We also use MATLAB's chol in the updated version SDPT3-2.3 of our matrix-based code.

For the solution of most second-order cone programming problems in DIMACS test set, however, MATLAB's chol routine is not competitive. This is largely due to the fact that the Schur complement matrix $M$ is often sparse for SOCPs and LPs, and MATLAB cannot sufficiently take advantage of this sparsity. To solve such problems more efficiently we imported the sparse Cholesky solver in Yin Zhang's LIPSOL [31], an interior-point code for linear programming problems. It should be noted that LIPSOL uses Fortran programs developed by Esmond Ng and Barry Peyton for sparse Cholesky factorization [18]. When SDPT3 uses LIPSOL's Cholesky solver, it first generates a symbolic factorization of the Schur complement matrix to determine the pivot order by examining the sparsity structure of this matrix carefully. Then, this pivot order is re-used in later iterations to compute the Cholesky factors. In contrast to the case of linear programming, however, the sparsity structure of the Schur complement matrix can change during the iterations for SOCP problems. If this happens, the pivot order has to be recomputed. We detect changes in the sparsity structure by monitoring the number of nonzero elements of the Schur complement matrix. Since the default initial iterates we use for an SOCP problem are unit vectors but subsequent iterates are not, there is always a change in the sparsity pattern of $M$ after the first iteration. After the second iteration, the sparsity pattern remains unchanged for most problems, and only one more change occurs in a small fraction of the test problems.

The effect of including a sparse Cholesky solver option for SOCP problems was dramatic. We observed speed-ups up to two orders of magnitude. SDPT3-3.0 automatically makes a choice between MATLAB's built-in chol routine and the sparse Cholesky solver based on the density of the Schur complement matrix.

\subsection{Vectorized matrices vs. sparse matrices}

The current release, SDPT3-3.0, of the code stores the constraint matrix in "vectorized" form as described in Sections 2 and 4. In the previous versions, and in SDPT3-2.3, A is a doubly subscripted cell array of symmetric matrices for the semidefinite blocks, as we outlined at the end of the previous section. The result of the change is that much less storage is required for the constraint matrix, and that we save a considerable amount of time in forming the Schur complement matrix $M$ in (11) by avoiding loops over the index $k$. Operations relating to forming and factorizing the Schur complement and hence computing the predictor search direction comprise much of the computational work for most problem classes, ranging from $25 \%$ for qpG11 up to $99 \%$ for the larger theta problems, the control problems, copo14, hamming-7-5-6, and the nb 


\begin{tabular}{|c|c|c|c|c|c|c|c|c|c|c|c|c|}
\hline & \multicolumn{6}{|c|}{ HKM } & \multicolumn{6}{|c|}{$\mathrm{NT}$} \\
\hline Problem & Itn & $\operatorname{err}_{1}$ & err $_{3}$ & err 5 & $\operatorname{err}_{6}$ & time & Itn & $\mathrm{err}_{1}$ & $\mathrm{err}_{3}$ & err 5 & $\operatorname{err}_{6}$ & time \\
\hline $\mathrm{bm} 1$ & 17 & $1-06$ & $3-13$ & $8-08$ & $1-07$ & 834 & 14 & $9-07$ & $5-13$ & $3-02$ & $3-02$ & 2891 \\
\hline copo14 & 12 & $2-11$ & $1-14$ & $8-10$ & 1-09 & 112 & 12 & $2-11$ & $9-15$ & $7-10$ & $1-09$ & 111 \\
\hline copo23 & 16 & $3-12$ & $6-14$ & $2-10$ & $4-10$ & 4375 & 16 & $2-12$ & $6-14$ & $2-10$ & $3-10$ & 4343 \\
\hline hamming-7-5-6 & 10 & $2-15$ & 0 & $2-10$ & $2-10$ & 83 & 10 & $3-15$ & 0 & $2-10$ & $2-10$ & 83 \\
\hline hamming-9-8 & 12 & $8-15$ & 0 & $2-10$ & $2-10$ & 341 & 12 & $7-15$ & 0 & $2-10$ & $2-10$ & 646 \\
\hline hinf12 & 42 & $2-08$ & $5-10$ & $-2-01$ & $1-08$ & 6 & 39 & $3-08$ & $3-10$ & $-2-01$ & $5-08$ & 7 \\
\hline hinf13 & 24 & $8-05$ & $1-12$ & $-2-02$ & $1-04$ & 4 & 23 & $8-05$ & $7-13$ & $-2-02$ & $9-05$ & 5 \\
\hline minphase & 32 & $1-08$ & 0 & $-2-04$ & $3-08$ & 6 & 37 & $2-08$ & 0 & $-4-04$ & $2-06$ & 9 \\
\hline torusg3-8 & 15 & $2-11$ & $8-16$ & 1-09 & 1-09 & 112 & 14 & $2-10$ & $7-16$ & $2-09$ & $2-09$ & 484 \\
\hline toruspm3-8-50 & 13 & $2-11$ & $6-16$ & $3-09$ & 3-09 & 93 & 14 & $5-11$ & $6-16$ & $2-10$ & $2-10$ & 470 \\
\hline truss 5 & 19 & $5-07$ & $6-15$ & $3-08$ & $2-07$ & 34 & 19 & $5-07$ & $1-14$ & $-9-08$ & $3-08$ & 37 \\
\hline truss8 & 22 & $3-06$ & $8-15$ & $-2-06$ & $2-07$ & 306 & 21 & $2-06$ & $1-14$ & $-8-09$ & $2-06$ & 299 \\
\hline
\end{tabular}

Table 3. Computational results on SDP problems in the DIMACS Challenge test set using SDPT3-2.3. These were performed on a Pentium III PC $(800 \mathrm{MHz})$ with $1 \mathrm{G}$ of memory.

problems. Other significant parts are computing the corrector search direction (up to $75 \%$ ) and computing step lengths (up to 60\%).

While we now store the constraint matrix in vectorized form, the parts of the iterates $\mathrm{X}$ and $\mathrm{Z}$ corresponding to semidefinite blocks are still stored as matrices, since that is how the user wants to access them.

Results are given in Tables 3 through 6: Tables 3 and 4 give results on the DIMACS problems for both SDPT3-3.0 and SDPT3-2.3, while Tables 5 and 6 give the comparable results for the SDPLIB problems. In all of these, the format is the same. We give the number of iterations required, the time in seconds, and four measures of the precision of the computed answer. These accuracy measures are computed as follows:

$$
\begin{aligned}
\operatorname{err}_{1} & =\frac{\|A x-b\|}{1+\max \left|b_{k}\right|}, \\
\operatorname{err}_{3} & =\frac{\left\|A^{T} y+z-c\right\|}{1+\max |c|}, \\
\operatorname{err}_{5} & =\frac{\langle c, x\rangle-b^{T} y}{1+|\langle c, x\rangle|+\left|b^{T} y\right|}, \\
\operatorname{err}_{6} & =\frac{\langle x, z\rangle}{1+|\langle c, x\rangle|+\left|b^{T} y\right|} .
\end{aligned}
$$

In err $_{3}$ the norm is subordinate to the inner product and the maximum is taken over all components of $c$. These measures are almost identical to the measures reported by Mittelmann in [15], except that he uses $\|b\|_{1}$ instead of $\max \left|b_{k}\right|$ in $\operatorname{err}_{1}$, and similarly $\|c\|_{1}$ instead of $\max |c|$ in $\operatorname{err}_{3}$. Mittelmann also reports cone violation measures $\mathrm{err}_{2}$ and err $_{4}$ which are always zero for our iterates.

In general, both versions of our codes solved all problems without secondorder cone constraints to reasonable accuracy (in terms of all measures) using either of the search directions. SDPT3-3.0 had difficulty obtaining high accuracy solutions to several DIMACS problems involving second-order cone constraints. 
R. H. Tütüncü, K. C. Toh,, M. J. Todd

\begin{tabular}{|c|c|c|c|c|c|c|c|c|c|c|c|c|}
\hline & \multicolumn{6}{|c|}{ HKM } & \multicolumn{6}{|c|}{$\mathrm{NT}$} \\
\hline Problem & Itn & err 1 & $\mathrm{err}_{3}$ & $\operatorname{err}_{5}$ & $\mathrm{err}_{6}$ & time & Itn & err $_{1}$ & $\mathrm{err}_{3}$ & err 5 & $\operatorname{err}_{6}$ & time \\
\hline$\overline{\mathrm{bm} 1}$ & 18 & $4-07$ & $8-12$ & $1-07$ & 1-07 & 811 & 16 & 1-08 & $5-13$ & $1-05$ & $1-05$ & 2758 \\
\hline copo14 & 15 & $1-10$ & $6-15$ & $-1-09$ & $8-10$ & 40 & 13 & $6-11$ & $6-15$ & 9-09 & 7-09 & 36 \\
\hline copo23 & 17 & $2-09$ & $1-14$ & $8-08$ & $5-10$ & 1805 & 16 & $8-10$ & $1-14$ & $2-08$ & $5-09$ & 1695 \\
\hline hamming-7-5-6 & 10 & $2-15$ & $9-15$ & $9-11$ & $9-11$ & 66 & 10 & $2-15$ & $9-15$ & $9-11$ & $9-11$ & 68 \\
\hline hamming-9-8 & 11 & $5-15$ & $9-14$ & 4-09 & $4-09$ & 212 & 11 & $5-15$ & $8-14$ & $4-09$ & $4-09$ & 418 \\
\hline hinf12 & 42 & $2-08$ & $4-10$ & $-2-01$ & $1-08$ & 5 & 39 & $2-08$ & $2-10$ & $-2-01$ & $6-09$ & 5 \\
\hline hinf13 & 23 & $9-05$ & $8-13$ & $-2-02$ & $3-05$ & 4 & 22 & $1-04$ & $9-13$ & $-2-02$ & $6-06$ & 4 \\
\hline minphase & 32 & $8-09$ & $3-12$ & $-2-04$ & $1-08$ & 5 & 37 & $2-08$ & $7-13$ & $-5-04$ & $3-06$ & 7 \\
\hline torusg3-8 & 15 & $2-11$ & $7-16$ & $3-10$ & $3-10$ & 89 & 14 & $2-10$ & $7-16$ & 3-09 & $3-09$ & 407 \\
\hline toruspm3-8-50 & 14 & $2-11$ & $6-16$ & $2-09$ & $2-09$ & 84 & 15 & $4-11$ & $6-16$ & $7-10$ & $7-10$ & 432 \\
\hline truss 5 & 16 & $4-07$ & $8-15$ & $-3-10$ & $1-07$ & 9 & 16 & $4-07$ & $8-15$ & $-1-07$ & $3-08$ & 10 \\
\hline truss 8 & 15 & $3-06$ & $8-15$ & $-3-06$ & $1-07$ & 44 & 14 & $2-06$ & $1-14$ & $5-07$ & $2-06$ & 47 \\
\hline filter48-socp & 38 & $\overline{11-06}$ & $\overline{9-14}$ & $1-05$ & $\overline{11-06}$ & 51 & 45 & $\overline{11-06}$ & $\overline{8-14}$ & $1-05$ & $\overline{11-06}$ & 60 \\
\hline filtinf1 & 27 & $3-05$ & $4-12$ & $2-01$ & $4-01$ & 38 & 27 & 3-05 & $2-11$ & $4-01$ & $7-01$ & 39 \\
\hline $\mathrm{nb}$ & 15 & $1-05$ & $2-09$ & $2-04$ & $2-04$ & 42 & 14 & $1-05$ & $1-08$ & $2-04$ & $2-04$ & 31 \\
\hline nb-L1 & 16 & $7-05$ & 4-09 & $2-05$ & $1-05$ & 73 & 16 & $2-04$ & $9-11$ & $1-06$ & $9-07$ & 59 \\
\hline nb-L2 & 12 & $2-09$ & $1-11$ & $6-09$ & $6-09$ & 57 & 11 & $4-09$ & $1-08$ & $5-07$ & $5-07$ & 45 \\
\hline nb-L2-bessel & 13 & $8-06$ & $4-12$ & $9-07$ & $5-08$ & 39 & 11 & $3-07$ & $2-09$ & $6-07$ & $7-07$ & 26 \\
\hline nql30 & 13 & $6-08$ & $5-09$ & $2-05$ & $4-05$ & 11 & 16 & $2-06$ & $3-11$ & $-5-06$ & $1-06$ & 12 \\
\hline nql60 & 13 & $4-07$ & $1-08$ & $4-05$ & $1-04$ & 63 & 15 & $3-06$ & $2-10$ & $-2-05$ & $9-06$ & 57 \\
\hline nql180 & 15 & $1-05$ & $3-08$ & $7-05$ & $1-03$ & 5622 & 16 & $7-05$ & $4-10$ & $-3-03$ & $6-05$ & 3235 \\
\hline nql30old & 12 & $5-05$ & $2-08$ & $-7-05$ & $1-04$ & 12 & 12 & $5-05$ & $2-08$ & $-8-05$ & $1-04$ & 12 \\
\hline nql60old & 13 & $1-04$ & 7-09 & $-8-04$ & $1-04$ & 87 & 13 & $9-05$ & $5-08$ & $-4-04$ & $5-04$ & 75 \\
\hline nql180old & 9 & $9-04$ & $3-05$ & $-5-02$ & $2-01$ & 4015 & 10 & $1-03$ & $8-06$ & $-3-01$ & $8-02$ & 2742 \\
\hline qssp30 & 21 & $7-08$ & 1-09 & $7-07$ & $8-07$ & 24 & 18 & $3-07$ & $2-11$ & $-1-07$ & $3-08$ & 17 \\
\hline qssp60 & 21 & $5-05$ & $2-09$ & $6-05$ & $2-05$ & 154 & 20 & $3-06$ & $1-11$ & $3-06$ & $1-07$ & 108 \\
\hline qssp180 & 24 & $3-04$ & $1-08$ & $8-04$ & $2-04$ & 17714 & 25 & $3-05$ & $4-12$ & $7-05$ & $4-08$ & 9790 \\
\hline qssp30old & 11 & $3-04$ & $4-05$ & $5-02$ & $6-02$ & 58 & 12 & $5-04$ & $6-05$ & $3-02$ & $4-02$ & 60 \\
\hline qssp60old & 11 & $4-04$ & $2-04$ & $1-01$ & $2-01$ & 397 & 12 & $4-04$ & $4-04$ & $1-01$ & $2-01$ & 382 \\
\hline sched-50-50-orig & 28 & $7-04$ & 3-09 & $-9-05$ & $6-06$ & 21 & 29 & $2-04$ & $3-07$ & $-1-05$ & $7-06$ & 20 \\
\hline sched-50-50-scaled & 23 & $1-04$ & $4-15$ & $2-05$ & $3-05$ & 18 & 22 & $6-05$ & $4-15$ & $1-06$ & $7-06$ & 16 \\
\hline sched-100-50-orig & 39 & $6-03$ & $3-11$ & $-8-04$ & $2-06$ & 63 & 33 & $6-03$ & $2-11$ & $8-04$ & $5-07$ & 50 \\
\hline sched-100-50-scaled & 26 & $8-04$ & $9-13$ & $1-04$ & $1-04$ & 44 & 22 & $7-04$ & $1-09$ & $3-04$ & $3-04$ & 35 \\
\hline sched-100-100-orig & 33 & $5-02$ & $1-10$ & $-2-02$ & $4-07$ & 102 & 50 & $5-01$ & $3-10$ & $1-00$ & $1-07$ & 136 \\
\hline sched-100-100-scaled & 19 & $4-02$ & $1-14$ & $-4-03$ & $3-06$ & 65 & 17 & $3-02$ & $1-14$ & $-2-03$ & $1-02$ & 55 \\
\hline sched-200-100-orig & 41 & $6-03$ & 3-09 & $-4-03$ & $3-06$ & 348 & 39 & $6-03$ & $1-08$ & $-5-03$ & $4-06$ & 309 \\
\hline sched-200-100-scaled & 27 & $3-03$ & 6-09 & $-8-04$ & 7-04 & 247 & 25 & 3-03 & $7-10$ & $-1-03$ & 3-04 & 216 \\
\hline
\end{tabular}

Table 4. Computational results on DIMACS Challenge problems using SDPT3-3.0. These were performed on a Pentium III PC $(800 \mathrm{MHz})$ with $1 \mathrm{G}$ of memory.

We comment on some of these problems in detail in Section 5.6. We note that on two problems, our codes terminated with an indication that $\mathrm{X}$ and $\mathrm{Z}$ were not both positive definite: qpG11 (version 2.3, NT only) and sched-100-100-orig (version 3.0, NT only). However, this is a conservative test designed to stop if numerical difficulties are imminent. Using SeDuMi's eigK.m routine to check the iterates, it was found that in both cases both variables were feasible in the conic constraints.

The objective values of the optimal solutions generated by our codes match the optimal objective values listed in Tables 1 and 2 in most cases. Exceptions, however, are not limited to the problems where our codes had accuracy problems. In particular, for problems bm1, torusg3-8, qap9, qap10, maxG51, and qpG51 SDPT3 generates accurate solutions whose optimal objective values dif- 


\begin{tabular}{|c|c|c|c|c|c|c|c|c|c|c|c|c|}
\hline & \multicolumn{6}{|c|}{ HKM } & \multicolumn{6}{|c|}{ NT } \\
\hline Problem & Itn & err 1 & $\mathrm{err}_{3}$ & $\operatorname{err}_{5}$ & $\mathrm{err}_{6}$ & time & Itn & $\mathrm{err}_{1}$ & $\mathrm{err}_{3}$ & $\operatorname{err}_{5}$ & $\operatorname{err}_{6}$ & time \\
\hline arch8 & 19 & $9-10$ & $5-13$ & $2-09$ & $2-09$ & $\overline{55}$ & 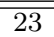 & 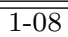 & $\overline{5-13}$ & $\overline{44-08}$ & 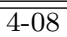 & $\overline{74}$ \\
\hline control7 & 23 & $7-07$ & $2-09$ & $1-07$ & $8-07$ & 149 & 22 & $4-07$ & 2-09 & $1-06$ & $2-06$ & 156 \\
\hline control10 & 24 & $6-07$ & 5-09 & $1-06$ & $3-06$ & 709 & 24 & $1-06$ & $6-09$ & $-1-06$ & $8-07$ & 802 \\
\hline control11 & 24 & $1-06$ & $6-09$ & $-3-06$ & $4-07$ & 1108 & 24 & $1-06$ & 6-09 & $-1-06$ & $1-06$ & 1245 \\
\hline gpp250-4 & 15 & $3-08$ & $5-14$ & $-9-09$ & 4-09 & 28 & 13 & 7-06 & $2-13$ & $1-05$ & $2-05$ & 62 \\
\hline gpp500-4 & 15 & $3-08$ & $2-14$ & 4-09 & 5-09 & 169 & 13 & 7-08 & $3-14$ & $2-05$ & $2-05$ & 501 \\
\hline hinf15 & 24 & $9-05$ & $2-12$ & $-4-02$ & $2-05$ & 7 & 23 & $1-04$ & $1-12$ & $-4-02$ & $2-04$ & 8 \\
\hline mcp250-1 & 13 & $2-11$ & $5-16$ & 4-09 & 4-09 & 14 & 15 & $7-12$ & $4-16$ & $9-10$ & $9-10$ & 55 \\
\hline mcp500-1 & 14 & $1-11$ & $5-16$ & $8-10$ & $8-10$ & 79 & 15 & $2-11$ & $5-16$ & 4-09 & 4-09 & 416 \\
\hline qap9 & 15 & $4-08$ & $3-13$ & $-2-05$ & $1-08$ & 19 & 15 & $5-08$ & $3-13$ & $-3-05$ & $1-08$ & 20 \\
\hline qap10 & 14 & $4-08$ & $3-13$ & $-6-05$ & $1-08$ & 34 & 14 & $4-08$ & $4-13$ & $-6-05$ & $5-09$ & 36 \\
\hline ss30 & 19 & $2-09$ & $2-13$ & 5-09 & 5-09 & 113 & 24 & $1-08$ & $2-13$ & $6-08$ & $6-08$ & 242 \\
\hline theta 3 & 14 & $3-11$ & $6-15$ & 1-09 & $1-09$ & 40 & 14 & $2-10$ & $6-15$ & $4-10$ & $2-10$ & 45 \\
\hline theta 4 & 15 & $2-10$ & $8-15$ & $2-09$ & $2-09$ & 160 & 15 & $4-10$ & $8-15$ & $4-10$ & $2-10$ & 175 \\
\hline theta5 & 15 & $2-10$ & $1-14$ & 2-09 & $2-09$ & 475 & 14 & $4-10$ & $1-14$ & 5-09 & 5-09 & 483 \\
\hline theta 6 & 15 & $5-10$ & $1-14$ & $-2-10$ & $4-10$ & 1224 & 15 & $5-10$ & $1-14$ & $1-10$ & $7-10$ & 1302 \\
\hline truss7 & 23 & $3-06$ & $2-13$ & $-5-06$ & $4-07$ & 6 & 22 & $4-06$ & $2-13$ & $-1-05$ & $2-08$ & 8 \\
\hline truss 8 & 22 & $3-06$ & $8-15$ & $-2-06$ & $2-07$ & 306 & 21 & $2-06$ & $8-15$ & $-8-09$ & $2-06$ & 299 \\
\hline equalG11 & 18 & $4-11$ & $3-16$ & $4-10$ & $4-10$ & 776 & 16 & $1-08$ & $3-16$ & $2-06$ & $2-06$ & 2451 \\
\hline equalG51 & 20 & 8-09 & $4-16$ & $-8-11$ & $2-10$ & 1586 & 20 & $5-08$ & $5-16$ & $2-08$ & $2-08$ & 5648 \\
\hline equalG32 & 19 & $1-10$ & $2-16$ & $2-09$ & $2-09$ & 10170 & 15 & $4-07$ & $2-16$ & $9-05$ & $9-05$ & 33618 \\
\hline $\max G 11$ & 14 & $2-11$ & $8-16$ & $2-09$ & $2-09$ & 292 & 14 & $4-11$ & $7-16$ & 1-09 & 1-09 & 1540 \\
\hline $\operatorname{maxG51}$ & 16 & $1-11$ & $4-16$ & $2-09$ & $2-09$ & 951 & 16 & $9-11$ & $3-16$ & $3-10$ & $3-10$ & 4171 \\
\hline $\max G 32$ & 15 & $1-10$ & $1-15$ & 2-09 & $2-09$ & 3726 & 15 & $2-10$ & $1-15$ & $6-10$ & $6-10$ & 24957 \\
\hline qpG11 & 14 & $1-11$ & 0 & 4-09 & 4-09 & 1429 & 15 & $2-11$ & 0 & 4-09 & 4-09 & 6789 \\
\hline qpG112 & 15 & $2-11$ & 0 & $4-10$ & $4-10$ & 337 & 15 & $6-11$ & 0 & $4-10$ & $4-10$ & 1693 \\
\hline qpG51 & 21 & $6-11$ & 0 & 4-09 & 4-09 & 4518 & 24 & 1-09 & 0 & 4-09 & $2-08$ & 19817 \\
\hline qpG512 & 17 & $2-10$ & 0 & 4-09 & 4-09 & 965 & 25 & $9-11$ & 0 & $2-09$ & $2-09$ & 6677 \\
\hline thetaG11 & 20 & 3-09 & $8-14$ & $-5-10$ & $2-10$ & 1196 & 17 & 8-09 & $5-15$ & 1-09 & 3-09 & 2699 \\
\hline thetaG11n & 15 & $4-12$ & 0 & $2-09$ & $2-09$ & 786 & 15 & $4-12$ & 0 & 1-09 & 1-09 & 2240 \\
\hline thetaG51 & 33 & $4-08$ & $7-13$ & 4-09 & 4-09 & 18992 & 30 & 7-09 & $8-14$ & $2-08$ & $2-08$ & 23851 \\
\hline thetaG51n & 19 & $2-09$ & $2-14$ & $-1-09$ & $1-08$ & 5159 & 22 & $5-09$ & $3-14$ & $-2-09$ & $7-10$ & 10415 \\
\hline
\end{tabular}

Table 5. Computational results on SDPLIB problems using SDPT3-2.3. These were performed on a Pentium III PC (800MHz) with $1 \mathrm{G}$ of memory.

fer from previously published optimal objective values which we believe to be incorrect. We also provide an accurate optimal objective value for equalG32 which was previously unavailable. Finally, we note that some of the listed optimal objective values in Table 1 contain sign errors, see the caption for Table 1 for details.

To compare the two codes in terms of time, we consider only the problems that both codes could solve, and omit the simplest problems with times under 20 seconds (the hinf problems, minphase, and truss5 and truss7). For the remaining problems, we compute the ratio of the times taken by the two codes, take its logarithm to base 2, and then plot the results in decreasing order of absolute values. The results are shown in Figures 1 and 2 for the HKM and NT search directions. A bar of height 1 indicates that SDPT3-3.0 was 2 times faster than SDPT3-2.3, of -1 the reverse, and of 3 that SDPT3-3.0 was 8 times faster. Note that the new version using vectorized matrices is almost uniformly faster and often at least $50 \%$ faster using either direction. 
R. H. Tütüncü, K. C. Toh,, M. J. Todd

\begin{tabular}{|c|c|c|c|c|c|c|c|c|c|c|c|c|}
\hline & \multicolumn{6}{|c|}{ HKM } & \multicolumn{6}{|c|}{$\mathrm{NT}$} \\
\hline Problem & Itn & err 1 & $\mathrm{err}_{3}$ & err. $_{5}$ & $\mathrm{err}_{6}$ & time & Itn & $\operatorname{err}_{1}$ & err $_{3}$ & err $_{5}$ & $\operatorname{err}_{6}$ & time \\
\hline $\operatorname{arch} 8$ & 21 & $1-09$ & $\overline{5-13}$ & $8-10$ & $8-10$ & 41 & 24 & $2-08$ & 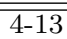 & $\overline{~ 1-07}$ & 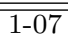 & $\overline{55}$ \\
\hline control7 & 22 & $5-07$ & $2-09$ & $8-07$ & $1-06$ & 111 & 22 & 7-07 & $2-09$ & $-1-07$ & $6-07$ & 129 \\
\hline control10 & 24 & $1-06$ & $6-09$ & $-1-06$ & $3-07$ & 508 & 24 & $1-06$ & 6-09 & $-1-06$ & 7-07 & 610 \\
\hline control11 & 24 & $2-06$ & $6-09$ & $-3-06$ & $3-07$ & 760 & 23 & $9-07$ & 6-09 & $-4-07$ & $2-06$ & 891 \\
\hline gpp250-4 & 15 & $8-08$ & $2-12$ & $-1-08$ & 4-09 & 24 & 13 & 7-08 & 6-14 & $3-06$ & $3-06$ & 55 \\
\hline gpp500-4 & 15 & $5-08$ & $1-12$ & $1-09$ & 3-09 & 152 & 18 & $3-08$ & $1-12$ & 5-09 & 7-09 & 601 \\
\hline hinf15 & 23 & $9-05$ & $2-12$ & $-4-02$ & $3-06$ & 6 & 22 & $1-04$ & $2-12$ & $-5-02$ & $2-05$ & 7 \\
\hline mcp250-1 & 14 & $3-12$ & $4-16$ & 1-09 & 1-09 & 12 & 15 & $1-11$ & $4-16$ & $2-10$ & $2-10$ & 40 \\
\hline mср500-1 & 15 & $1-11$ & $5-16$ & $6-10$ & $6-10$ & 60 & 16 & $3-11$ & $5-16$ & $2-10$ & $2-10$ & 327 \\
\hline qap9 & 15 & $4-08$ & $3-13$ & $-2-05$ & $1-08$ & 17 & 15 & $5-08$ & $3-13$ & $-3-05$ & $1-08$ & 18 \\
\hline qap10 & 14 & $4-08$ & $3-13$ & $-6-05$ & 9-09 & 30 & 13 & $4-08$ & $4-13$ & $-6-05$ & $6-08$ & 29 \\
\hline ss30 & 21 & $6-09$ & $3-13$ & $1-08$ & $1-08$ & 138 & 26 & 3-09 & $3-13$ & $1-07$ & $1-07$ & 264 \\
\hline theta3 & 15 & $2-10$ & $2-14$ & 1-09 & 1-09 & 37 & 14 & $2-10$ & $2-14$ & $4-10$ & $1-10$ & 39 \\
\hline theta 4 & 15 & $2-10$ & $3-14$ & 1-09 & 1-09 & 129 & 14 & $3-10$ & $3-14$ & $6-10$ & $4-10$ & 132 \\
\hline theta5 & 15 & $3-10$ & $4-14$ & $2-09$ & $2-09$ & 392 & 14 & $4-10$ & 4-14 & $3-10$ & $3-10$ & 398 \\
\hline theta 6 & 14 & $2-10$ & $5-14$ & 3-09 & 3-09 & 968 & 14 & $6-10$ & $5-14$ & $8-10$ & $1-09$ & 1028 \\
\hline truss7 & 23 & $3-06$ & $2-13$ & $-4-06$ & $3-07$ & 4 & 21 & $2-06$ & $1-13$ & $-2-06$ & 7-08 & 5 \\
\hline truss 8 & 15 & $3-06$ & $8-15$ & $-3-06$ & $1-07$ & 44 & 14 & $2-06$ & $1-14$ & 5-07 & $2-06$ & 47 \\
\hline "equalG11 & 17 & $2-10$ & $\overline{~ 4-16}$ & $\bar{~} 1-09$ & 1-09 & 611 & 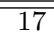 & $2-10$ & $7-15$ & 1-09 & $\overline{~ 1-09}$ & 2210 \\
\hline equalG51 & 20 & $2-08$ & $3-14$ & $6-10$ & $5-10$ & 1338 & 20 & $9-09$ & $5-16$ & $1-07$ & $1-07$ & 5050 \\
\hline equalG32 & 19 & $3-10$ & $2-16$ & 2-09 & $2-09$ & 8760 & 19 & $2-09$ & $4-15$ & 1-09 & 1-09 & 36683 \\
\hline $\max G 11$ & 15 & $9-12$ & $7-16$ & 5-09 & 5-09 & 190 & 15 & 4-11 & $7-16$ & $8-10$ & $8-10$ & 1357 \\
\hline $\max \mathrm{G} 51$ & 17 & $3-12$ & $2-16$ & $4-10$ & $4-10$ & 617 & 16 & $2-10$ & $3-16$ & $2-09$ & $2-09$ & 3077 \\
\hline $\operatorname{maxG} 32$ & 16 & $1-10$ & $1-15$ & 3-09 & 3-09 & 2417 & 16 & $2-10$ & $1-15$ & $6-10$ & $6-10$ & 23294 \\
\hline qpG11 & 16 & $2-11$ & 0 & $2-10$ & $2-10$ & 1514 & 15 & $1-10$ & 0 & 3-09 & 3-09 & 4528 \\
\hline qpG112 & 18 & $2-11$ & 0 & $2-10$ & $2-10$ & 225 & 17 & $5-11$ & 0 & 4-09 & 4-09 & 1532 \\
\hline qpG51 & 17 & $7-10$ & 0 & 3-09 & 3-09 & 3168 & 25 & $8-10$ & 0 & $4-09$ & $4-09$ & 15422 \\
\hline qpG512 & 19 & $4-10$ & 0 & $5-10$ & $5-10$ & 632 & 29 & $6-10$ & 0 & 4-09 & 4-09 & 5664 \\
\hline thetaG11 & 19 & $4-09$ & $1-13$ & $3-09$ & $2-09$ & 834 & 20 & $2-09$ & $2-14$ & $1-10$ & $5-10$ & 2334 \\
\hline thetaG11n & 15 & $1-12$ & $2-13$ & $4-10$ & $4-10$ & 456 & 15 & $1-12$ & $2-13$ & $4-10$ & $4-10$ & 1587 \\
\hline thetaG51 & 38 & $1-08$ & $3-13$ & $9-10$ & $1-09$ & 17692 & 30 & $2-08$ & $1-12$ & $3-08$ & $3-08$ & 18572 \\
\hline thetaG51n & 19 & $2-09$ & $5-13$ & $-2-09$ & 9-09 & 3921 & 23 & 3-09 & $5-13$ & $-1-08$ & $7-10$ & 8457 \\
\hline
\end{tabular}

Table 6. Computational results on SDPLIB problems using SDPT3-3.0. These were performed on a Pentium III PC $(800 \mathrm{MHz})$ with $1 \mathrm{G}$ of memory.

\subsection{HKM vs. NT}

The new version of the code allows only two search directions, HKM and NT. Version 2.3 also allows the AHO direction of Alizadeh, Haeberly, and Overton [2] and the GT (Gu-Toh) direction - see [23], but these are uncompetitive when the problems are of large scale. We intend to keep version 2.3 of the code available for those who wish to experiment with these other search directions, which tend to give more accurate results on smaller problems.

To compare the two remaining search directions, we again use bar charts to show their relative performance as in Figures 1 and 2. The behavior is significantly different depending on whether the problem has semidefinite blocks or not, so we report our results on two charts after categorizing the problems in this way. From Figure 3, it is clear that the HKM direction is almost universally faster than the NT direction for problems with semidefinite blocks. NT offers a slight advantage on only two problems, copo14 and copo23, both with many 


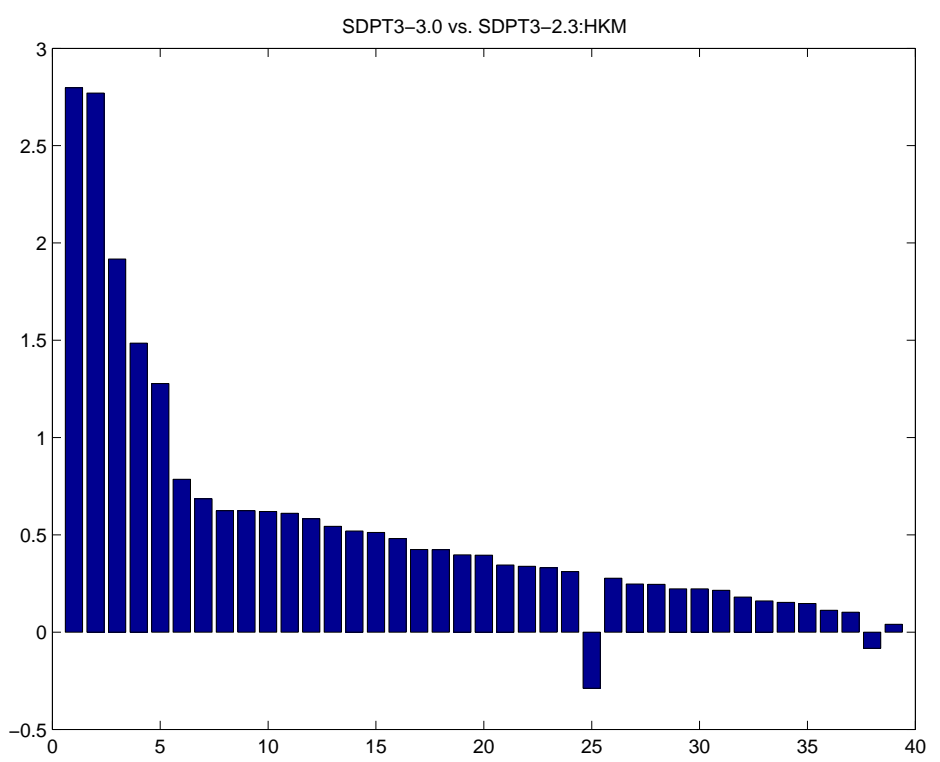

Figure 1. Comparing SDPT3-3.0 and SDPT3-2.3 using the HKM search direction. Bars above the axis demonstrate a win for 3.0.

small blocks. The HKM direction is much faster on maxG32 and considerably faster on the other maxG and the qpG problems.

Figure 4 shows a reversed pattern for problems with quadratic blocks and no semidefinite blocks. The NT direction was faster on all but three of such problems. The reason for this behavior is not hard to understand. By comparing the formula in (23) for the HKM direction with (29) for the NT direction, it is clear that more computation is required to assemble the Schur complement matrix and more low-rank updating is necessary for the former direction, and these computations can dominate the total work in each iteration. Because there is a class of problems on which the NT direction is faster, we feel it is worthwhile to keep both options.

\subsection{Homogeneous vs infeasible interior-point methods}

Version 2.3 also allows the user to employ homogeneous self-dual algorithms instead of the usual infeasible interior-point methods. However, this option almost always took longer than the default choice, and so it has been omitted from the current release. One theoretical advantage of the homogeneous selfdual approach is that it is oriented towards either producing optimal primal and dual solutions or generating a certificate of primal or dual infeasibility, while the infeasible methods strive for optimal solutions only, but detect infeasibility if either the dual or primal iterates diverge. However, in our limited testing on randomly generated infeasible problems, we have observed no advantage to 


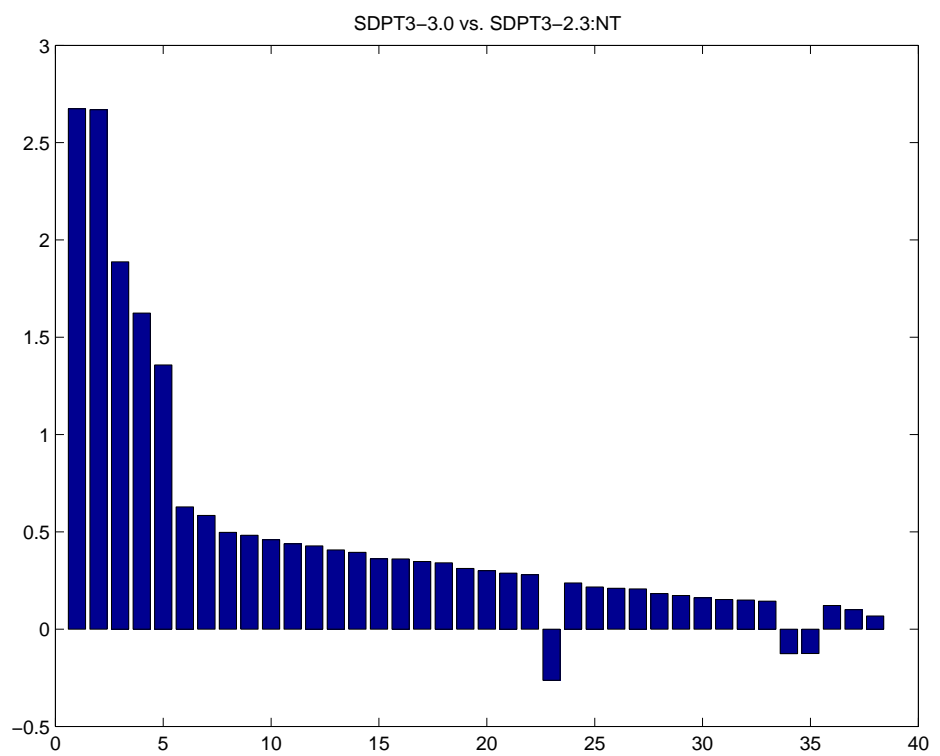

Figure 2. Comparing SDPT3-3.0 and SDPT3-2.3 using the NT search direction. Bars above the axis demonstrate a win for 3.0.

the homogeneous methods in detecting infeasibity. We should mention, however, that SDPT3-3.0 does not detect infeasibility in the problem filtinf1, but instead stops with a primal near-feasible solution and a dual feasible solution when it encounters numerical problems.

\subsection{Presentation of problems}

We note that qpG11 and qpG112, and similarly qpG51 and qpG512, are basically the same problem, but in the second version the linear variables are explicitly identified, rather than being part of a large sparse semidefinite block. The improvement in running time is dramatic: a factor of three to five. It is thus crucial to present problems to the algorithms correctly. Unfortunately the versions of qpG11 and qpG51 in SDPLIB do not show this structure explicitly. SDPT3-3.0 provides a preprocessor routine called detect_diag.m that can be executed to detect such structures.

We also remark that the computation of the Lovasz theta function for a graph can be expressed as a semidefinite programming problem in two ways, and one of these is much more compact than the other, requiring a linear constraint only for each edge of the graph rather than also for each node, and so the problems thetaG11n and thetaG51n are much easier to solve than thetaG11 and thetaG51, here by a factor up to three, although they are alternative semidefinite formulations of the same graph-theoretic problem for the same graphs. 


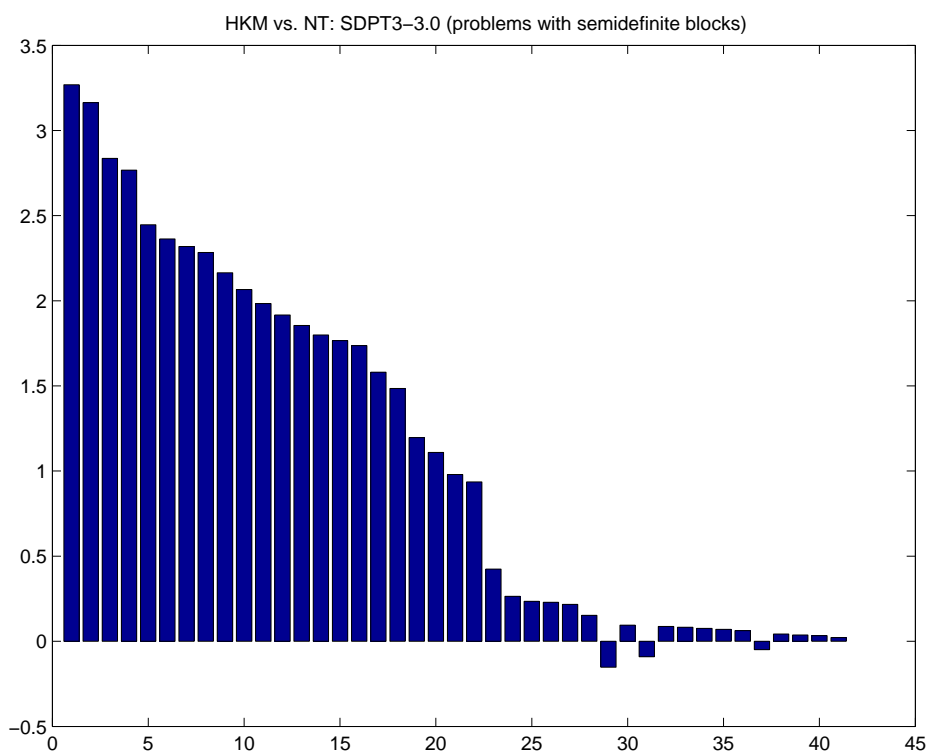

Figure 3. Comparing the HKM and NT search directions in SDPT3-3.0 for problems that have semidefinite blocks. Bars above the axis demonstrate a win for the HKM direction.

Finally, SDPT3-2.3 includes specialized routines to compute the Schur complement matrices directly for certain classes of problem (e.g., maxcut problems). In earlier versions of SDPT3, these specialized routines had produced dramatic decreases in solution times, but for SDPT3-2.3, these gains are marginal, since our general sparse matrix routines provide almost as much speedup. We have therefore dropped these routines in SDPT3-3.0.

\subsection{Accuracy problems}

The accuracy of the solutions obtained by SDPT3-3.0 on several of the problems with second-order cone constraints is less than ideal. While our current implementation may have inherent limitations to solve such problems with high accuracy, the structure of some of these problems contribute to the accuracy problems SDPT3-3.0 faced on them. Many of these problems, including the problems from the antenna set (nb, nb-L1, nb-L2, and nb-L2-bessel), from the nql set (nq130, nq130old, etc.) and the qssp set (qssp30, qssp30old, etc.), are reformulations of problems with variables that are unrestricted in sign. In these problems, free variables are represented as the difference of two nonnegative variables to fit the problem into the standard form conic optimization framework. This transformation has some well-known unpleasant consequences for interiorpoint algorithms. The primal optimal face is unbounded (when nonempty), the dual feasible set does not have an interior, and there is no central path. For most of these problems, SDPT3-3.0 generates diverging iterates and this creates nu- 


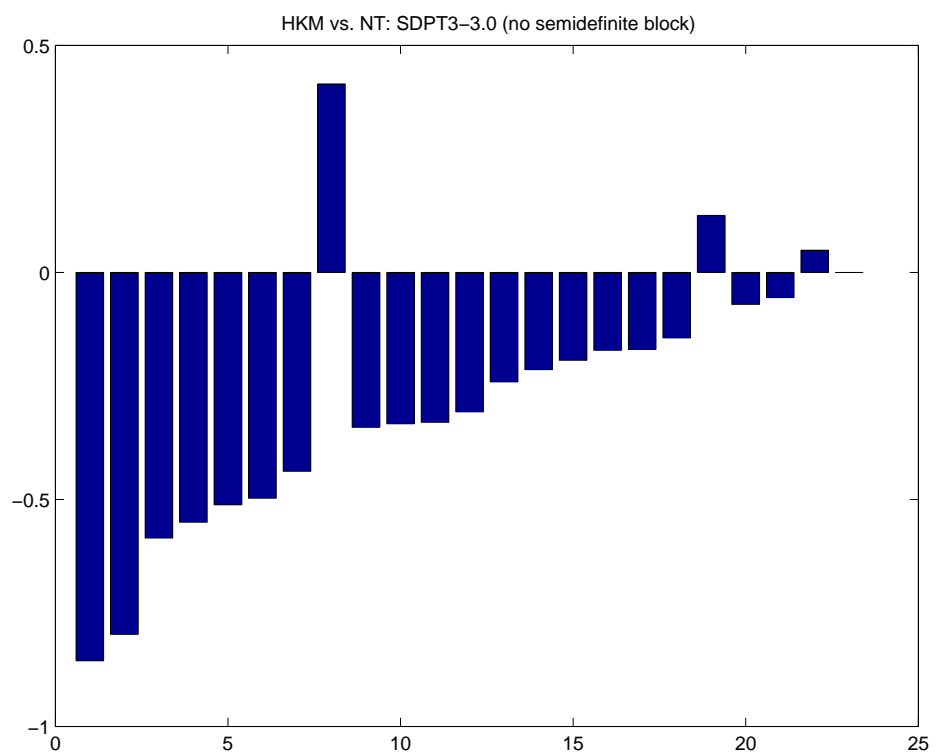

Figure 4. Comparing the HKM and NT search directions in SDPT3-3.0 for problems with quadratic blocks and no semidefinite blocks. Bars below the axis demonstrate a win for the NT direction.

merical difficulties as the algorithm progresses. Preliminary experiments showed that free-variable handling techniques that are commonly used in LP solvers can alleviate many of the accuracy problems we observed and such techniques will be included in future releases of our code.

\section{Conclusion}

Linear optimization problems involving cone constraints (e.g., positive semidefiniteness of variable matrices) continue to arise in a wide array of applications ranging from engineering and control to combinatorial optimization and scheduling. This paper describes computational experiments with a new version of SDPT3, a MATLAB implementation of infeasible-start primal-dual path following algorithms for the solution of such problems. Our experiments indicate that SDPT3 is a very robust code for solving medium-sized semidefinite optimization problems accurately and efficiently. The new version of SDPT3 is significantly faster than the previous versions 2.2 and 2.3. We also observe that, when using the HKM direction one can often obtain similar quality solutions for SDPs two-to-three times faster than when one uses the NT direction.

For problems with second-order (quadratic) cone constraints, experiments indicate that there is room for improvement in SDPT3 - especially with regards to the accuracy of the generated solutions. The efficiency of the two types of search directions we use is reversed for SOCPs: Similar quality solutions can often be obtained faster using the NT direction rather than the HKM direction. 
Overall, SDPT3 finds its niche as an efficient high-quality solver for medium sized semidefinite optimization problems.

\section{References}

1. F. Alizadeh, Interior point methods in semidefinite programming with applications to combinatorial optimization, SIAM J. Optim., 5 (1995), pp. 13-51.

2. F. Alizadeh, J.-P.A. Haeberly, and M.L. Overton, Primal-dual interior-point methods for semidefinite programming: convergence results, stability and numerical results, SIAM J. Optim., 8 (1998), pp. 746-768.

3. F. Alizadeh, J.-P.A. Haeberly, M.V. Nayakkankuppam, M.L. Overton, and S. Schmieta, SDPPACK user's guide, Technical Report, Computer Science Department, NYU, New York, June 1997.

4. B. Borchers, SDPLIB 1.2, a library of semidefinite programming test problems, Optim. Methods Softw., 11 \& 12 (1999), pp. 683-690. Available at http://www.nmt.edu/ borchers/sdplib.html.

5. B. Borchers, CSDP, a C library for semidefinite programming, Optim. Methods Softw., 11 \& 12 (1999), pp. 613-623. Available at http://www.nmt.edu/ borchers/csdp.html.

6. K. Fujisawa, M. Kojima, and K. Nakata, Exploiting sparsity in primal-dual interior-point method for semidefinite programming, Math. Program., 79 (1997), pp. 235-253.

7. K. Fujisawa, M. Kojima, and K. Nakata, SDPA (semidefinite programming algorithm) - user's manual, Research Report, Department of Mathematical and Computing Science, Tokyo Institute of Technology, Tokyo. Available via anonymous ftp at ftp.is.titech.ac.jp in pub/OpRes/software/SDPA.

8. D. Goldfarb, and K. Scheinberg, A product-form Cholesky factorization implementation of an interior-point method for second order cone programming, preprint.

9. G.H. Golub, and C.F. Van Loan, Matrix Computations, 2nd ed., (Johns Hopkins University Press, Baltimore, MD, 1989)

10. C. Helmberg, F. Rendl, R. Vanderbei and H. Wolkowicz, An interior-point method for semidefinite programming, SIAM J. Optim., 6 (1996), pp. 342-361.

11. N.J. Higham, Accuracy and Stability of Numerical Algorithms, (SIAM, Philadelphia, 1996)

12. M. Kojima, S. Shindoh, and S. Hara, Interior-point methods for the monotone linear complementarity problem in symmetric matrices, SIAM J. Optim., 7 (1997), pp. 86-125.

13. The MathWorks, Inc., Using MATLAB, (The MathWorks, Inc., Natick, MA, 1997)

14. S. Mehrotra, On the implementation of a primal-dual interior point method, SIAM J. Optim., 2 (1992), pp. 575-601.

15. H.D. Mittelmann, An independent benchmarking of SDP and SOCP solvers, Math. Program., this issue.

16. R.D.C. Monteiro, Primal-dual path-following algorithms for semidefinite programming, SIAM J. Optim., 7 (1997), pp. 663-678.

17. Yu. Nesterov and M. J. Todd, Self-scaled barriers and interior-point methods in convex programming, Math. Oper. Res., 22 (1997), pp. 1-42.

18. E.G. Ng and B.W. Peyton, Block sparse Cholesky algorithms on advanced uniprocessor computers, SIAM J. Sci. Comput., 14 (1993), pp. 1034-1056.

19. G. Pataki and S. Schmieta, The DIMACS library of mixed semidefinite-quadratic-linear programs. Available at http://dimacs.rutgers.edu/Challenges/Seventh/Instances.

20. F.A. Potra and R. Sheng, On homogeneous interior-point algorithms for semidefinite programming, Optim. Methods Softw. 9 (1998), pp. 161-184.

21. J.F. Sturm, Using SeDuMi 1.02, a Matlab toolbox for optimization over symmetric cones, Optim. Methods Softw., 11 \& 12 (1999), pp. 625-653.

22. M.J. Todd, K.C. Toh, R.H. Tütüncü, On the Nesterov-Todd direction in semidefinite programming, SIAM J. Optim., 8 (1998), pp. 769-796.

23. K. C. Toh, Some new search directions for primal-dual interior point methods in semidefinite programming, SIAM J. Optim., 11 (2000), pp. 223-242.

24. K.C. Toh, A note on the calculation of step-lengths in interior-point methods for semidefinite programming, Comput. Optim. Appl., 21 (2002), pp. 301-310.

25. K.C. Toh, M.J. Todd, R.H. Tütüncü, SDPT3 - a Matlab software package for semidefinite programming, Optim. Methods Softw., 11 \& 12 (1999), pp. 545-581. 
26. T. Tsuchiya, A polynomial primal-dual path-following algorithm for second-order cone programming, Technical Report, The Institute of Statistical Mathematics, Minato-Ku, Tokyo, October 1997.

27. T. Tsuchiya, A convergence analysis of the scaling-invariant primal-dual path-following algorithms for second-order cone programming, Optim. Methods Softw., 11 \& 12 (1999), pp. $141-182$.

28. R.H. Tütüncü, K.C. Toh, M.J. Todd, SDPT3 - a Matlab software package for semidefinite-quadratic-linear programming, version 3.0. Available at http://www.math.nus.edu.sg/ mattohkc/.

29. L. Vandenberghe and S. Boyd, Semidefinite programming, SIAM Rev., 38 (1996), pp. 49-95.

30. L. Vandenberghe and S. Boyd, User's guide to SP: software for semidefinite programming, Information Systems Laboratory, Stanford University, November 1994. Available via anonymous ftp at isl.stanford.edu in pub/boyd/semidef_prog. Beta version.

31. Y. Zhang, Solving large-scale linear programs by interior-point methods under the MATLAB environment, Optim. Methods Softw., 10 (1998), pp. 1-31. 